\title{
The Step Response of Left Ventricular Pressure to Ejection Flow: A System Oriented Approach
}

\author{
Herman B.K. Boom and Hessel Wijkstra* \\ Division of Biomedical Engineering \\ Department of Electrical Engineering \\ University of Twente \\ 7500 AE Enschede \\ The Netherlands \\ * Department of Urology \\ Radboud University Hospital \\ $6500 \mathrm{HB}$ Nijmegen \\ The Netherlands \\ (Received 4/25/91)
}

\begin{abstract}
Left ventricular pressure is dependent on both ventricular volume and ventricular ejection flow. These dependencies are usually expressed by ventricular elastance, and resistance, respectively. Resistance is a one-valued effect only, when ejection flow either is constant or increases. Decreasing ejection flow elicits a third effect: a decrease of elastance. The effects of elastance, resistance and elastance depression were modeled in a three-compartment model consisting of a dead-volume compartment, an elastance compartment, and a second series-elastance compartment connected to the elastance compartment by a resistance. This model was identified with the pressure response determined experimentally by imposing pumped constant-flow ejection epochs on isolated rabbit hearts. The experimental flow epochs consisted of two phases of constant flow separated by an increasing or decreasing flow step. It was found that elastance is not changed after the flow step if this is positive or zero. Negative flow steps induced a deactivation of elastance that is linearly dependent on the difference between isovolumic pressure that would be developed at the volume existing at the time of measurement and actual pressure. The parameters found from the identification procedure are ventricular active volume, nondepressed elastance, series-elastance, resistance, and the elastance deactivation factor. The first four parameter values were found in agreement with other results reported in literature. The elastance depression factor is a new parameter that could be of physiological or clinical significance since it may be related to the inability of the force generators in the heart muscle to be restored to their full number, after being inactivated or decoupled by filament sliding associated with ejection. On the basis of the results, an alinear state-model of the ventricle, for arbitrary, including physiological flow patterns is proposed.
\end{abstract}

Keywords-Ventricular elastance, Resistance, Ventricular state models.

Acknowledgments - This work was supported by The Netherlands Organization for Scientific Research (NWO).

Address correspondence to Herman B.K. Boom, Division of Biomedical Engineering, Department of Electrical Engineering, University of Twente, 7500 AE Enschede, The Netherlands. 


\section{INTRODUCTION}

In the last decades much effort has been invested in the search for ventricular indices that could represent the pumping quality of the left ventricle $(24,107)$. Such indices reflect a state, usually called the contractile state of the ventricle. Several lines of research were followed. In one it was realized that ventricular state variables reflect a corresponding state of the ventricular myocardium in its interaction with the complex and variable geometry of the ventricle. A number of papers have been published $(6,10,17,70,94,111)$ describing models of ventricular pressure generation based on a detailed inventory of cardiac muscle mechanics and ventricular anatomy. Global and measurable ventricular phenomena could thus be described; but no useful new definitions of ventricular performance arose from them. One reason is that not only should an index merely be defined by the model, but it also should be possible to actually derive it from physiological or clinical measurements. This would imply an inverse procedure with the detailed physiological model under study. Inverse procedures tend to be unstable if the model used is complicated.

An alternative approach, therefore, has been to start with the physiological measurements proper, using models derived from the data. Campbell et al. (21) discuss the interaction between the ventricle and the loading arterial tree on the basis of "global" concepts like elastance and resistance. They could thus define a ventricular state equation as part of a state model that could be identified from experiments.

Such models can very easily be made complex and could accurately describe ventricular phenomena. However, there is a general agreement that such models should be as simple as possible in order to optimally compress information present in the measurements into useful empirical relationships $(2,5,7,14,20,25,26,29,48,85,87,108$, 109). This parametrization, which has a long history $(39,82,100)$, has led to the proposal of a number of contractility indices $(3,40,63,68)$, several of which enjoyed some physiological and even clinical popularity for some time.

An index widely accepted is ventricular elastance, introduced by Suga, 1969 (93), who concluded a longer history of investigations $(67,100)$. Its usefulness is based on the fact that under certain restrictions, during ventricular ejection, there is a unique, instantaneous, nearly linear relation between ventricular volume and ventricular pressure (95); according to the equation:

$$
P(t)=e \cdot f(t) \cdot\left[B(t)-B_{d}\right]
$$

where $P(t)$ is ventricular pressure as a function of time, $B(t)$ is ventricular volume, and $B_{d}$ is a constant. $e \cdot f(t)=E(t)$ is ventricular elastance. It was demonstrated that the value of $e$ did not change if the contractile conditions within the myocardial muscle did not change. Also, $e$ increased and decreased with positive and negative inotropic interventions (95).

In engineering terms, (1) can be considered as the definition of a ventricular state model (Fig. 1a). The state vector consists of one component, $e$. Input is $B(t)$, output is normalized pressure $P(t) / f(t)$. In this way, clinically determining elastance is a system identification process. Simple, even trivial as this system is, it is not linear, since the output is not a linear combination of the components of the state vector and the input vector.

Global and empirical models have a limited area of validity. One way of dealing with deviations encountered experimentally consists of defining additional state vec- 


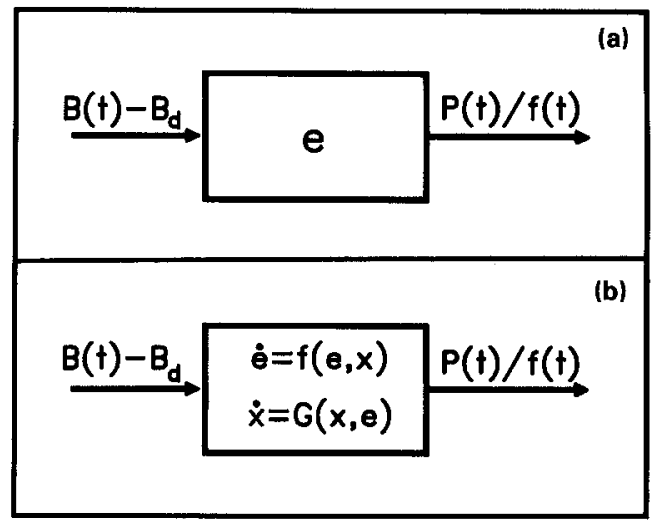

FIGURE 1. Ventricular state models. The ventricle is designated by a black box, in which one or more variables define the state. Input: $B_{t}(t)-B_{d}$ : "active" volume; $B_{d}$ : "dead volume". Output: $p(t) / f(t)$ : time normalized pressure depends on both state and input. Panel (a) Rudimentary state model containing one state variable $e$ which is constant $(e=0)$ during systole. Output given by: $p(t) / f(t)=$ $e \cdot\left[B_{t}(t)-B_{d}\right]$. Since the output is a multiplication of state variable and input $B_{t}-B_{d}$, this trivial model is nevertheless alinear. Panel (b) Extended ventricular state model. Elastance $e$ is not constant during systole, satisfying a differential equation to be found from the present study. $X$ : one or more additional state variables to be derived from experimental data.

tor components and thus making the model more accurate. As to the elastance model, it was shown in isolated ventricles (97), as well as in the intact animal $(8,20,28,58,91)$, that deviations occur for greater stroke volumes and ejection fractions. This was also shown for extreme conditions on contractile state by Burkhoff et al. (19).

Subsequent work showed that apart from ventricular elastance at least the following effects can be discerned on ventricular pressure during the systolic ejection phase:

(a) During ejection, pressure is attenuated by an amount related to flow intensity being pumped out, and thus to the rate of ventricular volume decrease. This was shown by Campbell et al. (20), Hunter et al. (47), Shroff et al. (87), and Vaartjes and Boom (102). So next to elastance also a resistance is operative in the ventricular model.

(b) Stepwise increasing ejection flow was shown by Boom (13), Campbell et al. (23), Covell et al. (27), Schiereck and Boom $(85,86)$, Templeton et al. (99), Vaartjes and Boom (102), and Yasumura et al. (112) to cause a lag in the corresponding pressure drop. This was compatible with the presence of a second, stiffer elastance which appears in series with the primary elastance.

(c) In addition to (b) stepwise decreasing flow exerts an effect on pressure comparable to a decrease of elastance as demonstrated by Boom and Wijkstra (15), and Wijkstra and Boom $(109,110)$.

Analyzing the relationship between ventricular flow and pressure has proved to be complicated by the understandable inclination of researchers to use physiologic flow patterns. Thus, the influence of flow changes, and especially the difference between increasing and decreasing flow, was obscured. Vaartjes and Boom (102), and later Wijkstra and Boom (109) used constant flow pulses in analogy with the constant volume situation, which in the past clarified basic phenomena in the generation of ven- 
tricular pressure (39). In particular, in studying what happens at the start and at the end of the constant flow epochs, that is, by studying the flow step response, the findings of (b) and (c) arose.

For a linear system the flow step response would suffice to derive the transfer function of the system from flow to pressure. However, the response for positive and negative flow steps was not symmetrical, showing that the system is not linear. The present paper starts from the concept that physiological flow can be thought of as a succession of infinitesimally small flow steps. Such steps differ from those used for (b) and (c) in that they do not step from relatively large flow to zero or vice versa. Therefore, the response of pressure to constant flow epochs, stepping from one value to another, is studied. On the basis of the results, it will be proposed how the state model of Fig. $1 \mathrm{~b}$ can be expanded in order to arrive at a model that quantitatively describes pressure as a function of time for various volume and, hence, flow-time functions.

\section{THEORY}

Use will be made of a three-compartment model (Fig. 2). Total ventricular volume $B_{t}$ is assumed to be composed of three compartments. One, the elastance compartment, with volume $B_{e}$ obeys

$$
P_{e}(t)=E_{e}(t) \cdot B_{e}=\epsilon \cdot e \cdot B_{e}(t) \cdot f(t)
$$

where $P_{e}$ is pressure in the elastance compartment. Constant $e$ is a scale factor of elastance allowing $f(t)$ to have amplitude 1. Decrease of elastance, as it occurs during ejection, is indicated by $\epsilon<1$. The time function $f(t)$ has the same meaning as in Eq. 1.

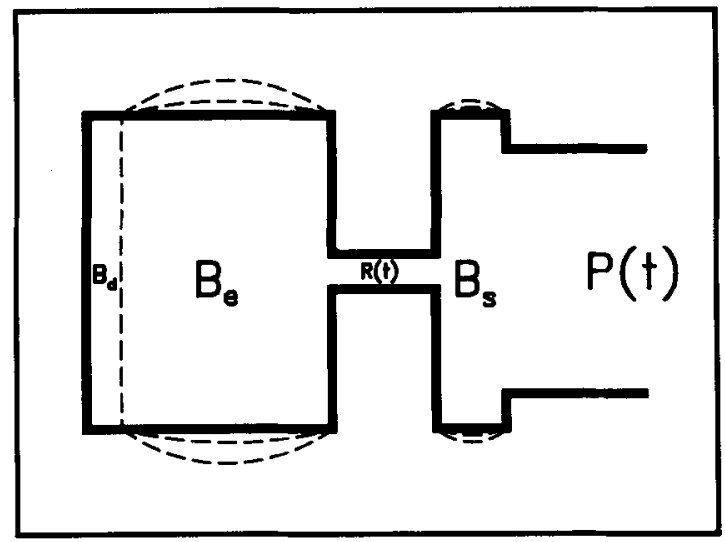

FIGURE 2. Three compartment model of the left ventricle. An active elastant compartment, volume $B_{\theta}$, elastance $E_{a}(t)$, combined with a constant "dead" volume compartment, volume $B_{d}$, is connected to a stiffer series-elastant compartment, volume $B_{s}$, elastance $E_{s}(t)$, by way of a Resistance $R(t)$. The series-elastant compartment communicates freely with the output. Therefore, output pressure $P(t)$ is equal to series-elastant pressure. During ejection, flow through $R(t)$ causes a pressure difference resulting in elastant pressure $P_{Q}$ to be higher than $P(t)$. During isovolumic contraction the pressure difference between $B_{e}$ and $B_{s}$ can be equilibrated. Elastances and resistance are all time variable in constant mutual proportions: $\epsilon \cdot R(t)=\mu \cdot E_{\theta}(t)$, and $\epsilon \cdot E_{s}(t)=\sigma \cdot E_{\theta}(t)$. 
A dead volume compartment as indicated in Eq. 1 is represented by a compartment with volume $B_{d}$. A third compartment, the series-elastic, communicates freely with the output of the model and has a volume $B_{s}$ according to

$$
P(t)=\sigma \cdot e \cdot B_{s}(t) \cdot f(t)=\sigma \cdot e \cdot\left(B_{t}-B_{e}-B_{d}\right) \cdot f(t)
$$

where $P(t)$ denotes ventricular output pressure. According to Schiereck and Boom (85), $\sigma \gg 1$ so that the series-elastic compartment is appreciably stiffer than the primary elastic compartment. During isovolumic contraction, compartment $B_{s}$ can be in equilibrium with $B_{e}: P_{e}=P_{s}$, or, $\sigma \cdot B_{s}=\epsilon \cdot B_{e}$. In that case no flow occurs through the connection between the compartments. Therefore, flow introduces an additional pressure difference between the compartments given by

$$
P(t)-P_{e}(t)=R(t) \cdot \frac{d B_{e}}{d t}=\mu \cdot e \cdot \frac{d B_{e}}{d t} \cdot f(t)
$$

where $R(t)$ is resistance, which similarly to series-elastance has been found to be instantaneously proportional to $E_{e}(87,101,102)$. Quantities $\sigma$ and $\mu$ are thus constants.

Isovolumic (preejection) pressure $P_{\text {iso }}(t)$ is given by

$$
P_{\mathrm{iso}}(t)=\frac{\sigma \cdot \epsilon}{\sigma+\epsilon} \cdot e \cdot\left(B_{t}-B_{d}\right) \cdot f(t)
$$

As can be easily found, by substituting Eqs. 2 and 3 into Eq. $4, B_{e}$ is subject to the differential equation

$$
\dot{B}_{e}+\frac{\epsilon+\sigma}{\mu} \cdot B_{e}=\frac{\sigma}{\mu} \cdot\left(B_{t}-B_{d}\right)
$$

which shows that the primary state model of the ventricle is expanded by $B_{e}$ which also becomes a state variable.

For the case that $\epsilon$ is a constant (which will be shown to be true for constant or increasing flow), and $B_{t}(t)$ is a linear function of time (constant flow), Eq. 6 can be solved straightforwardly. For instance, in Laplace representation, if $B_{t}(0)$ is ventricular volume at the start of ejection, and $\phi$ (constant) is ejection flow; for the constant flow epoch

$$
b_{t}(s)=\frac{B_{t}(0)}{s}-\frac{\phi}{s^{2}}
$$

Thus

$$
b_{e}(s)=\frac{s^{2} \cdot B_{e}(0)+s \cdot \omega_{s} \cdot\left\{B_{t}(0)-B_{d}\right\}-\omega_{s} \cdot \phi}{s^{2} \cdot\left(s+\omega_{t}\right)}
$$

where small letters imply variables in the Laplace domain and $s$ is the Laplace variable. Frequencies $\omega_{s}$ and $\omega_{t}$ are reciprocal time constants given by 


$$
\omega_{s}=\frac{\sigma}{\mu} \quad \text { and } \quad \omega_{t}=\frac{\sigma+\epsilon}{\mu}
$$

respectively. The three terms on the right side of Eq. 8 represent a negative exponential, an ascending exponential, and an ascending exponential plus a decreasing linear function, respectively. The linear function corresponds to the linear decrease of volume as results from constant ejection flow.

The Laplace transform of output variable $P(t) / f(t)$ is found from

$$
£\left[\frac{P(t)}{f(t)}\right]=\sigma\left(b_{t}-b_{e}-\frac{B_{d}}{s}\right) \cdot e
$$

where $£[F(t)]$ denotes the Laplace transform of function $F(t)$.

Ventricular pressure can be calculated by substituting suitable starting conditions for $B_{t}(0)$ and $B_{e}(0)$ into Eq. 8, and the result for $b_{e}(s)$ into Eq. 10. We are interested in the response of pressure to flow steps from one value to another. Such steps occur at $t_{1}$ in Fig. 3. In this figure the end of the isovolumic contraction phase is at $t_{0}$. Thus, taking first $t_{0}=0$

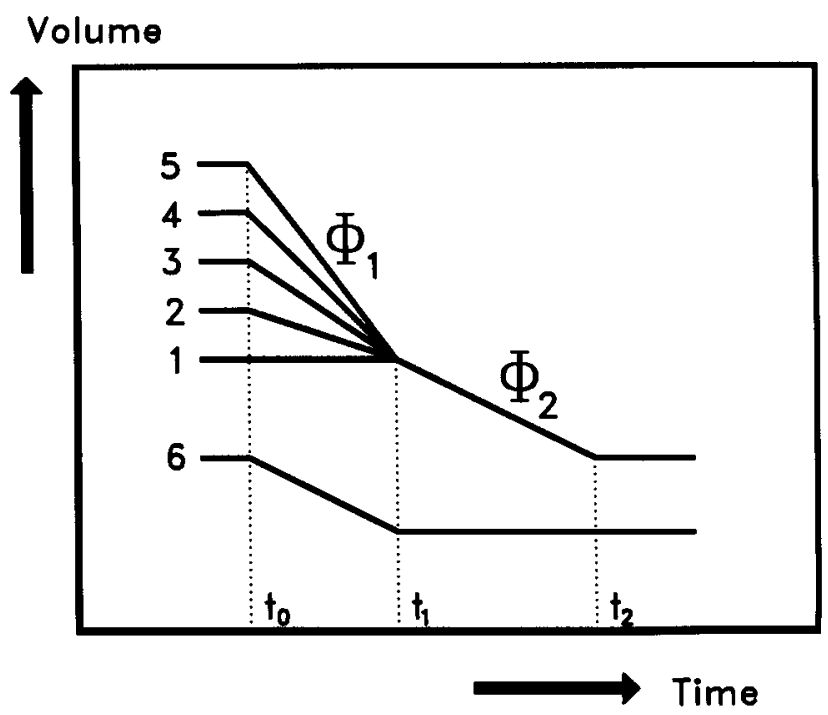

FIGURE 3. Volume time courses during pumped flow interventions on the ventricle. The epochs between either $t_{0}$ and $t_{1}$ or $t_{1}$ and $t_{2}$, uniformly decrease volumes and thus, imply constant ejection. From $t_{1}$ until $t_{2}$, trajectories 1 through 5 , have identical time courses. Any difference in developed pressure during this interval is uninfluenced by volume, flow or time differences. Volume-time course 2: positive flow step at $t_{1}$. Volume-time courses 4 and 5 : negative step $t_{1}$. Volume-time course 3: $\phi_{1}=\phi_{2}$, no flow switching. Volume-time course 1: $\phi_{1}=0$. Volume-time course 6: $\phi_{2}=0$. Courses 1 and 6 single are constant flow epochs. Equation 13 describes pressure development after a flow step at which $t=0$ is defined. Since the equation is invariant for starting time it can be applied at either $t_{0}$ or $t_{1}$. Application of Eq. 13 with $t_{0}=0$ at volume-time courses 2 through 5 yields a description of the pressure response during a constant flow epoch. Application with $t_{1}=0$ at time course 6 $\left(\phi_{2}=0\right)$ likewise describes the pressure response following a constant flow epoch. 


$$
B_{e}(0)=\frac{\sigma}{\sigma+\epsilon}\left\{B_{t}(0)-B_{d}\right\}
$$

This is substituted into Eq. 8 after this equation has been transformed back to the time domain. Thus, the volume of the elastic compartment at $t_{1}$, the time of the flow step in Fig. 4b, is

$$
B_{e}\left(t_{1}\right)=\frac{\sigma}{\epsilon_{1}+\sigma} \cdot\left\{B_{t}\left(t_{0}\right)-B_{d}\right\}+\frac{1}{\omega_{t 1}}\left[\frac{\omega_{s}}{\omega_{t 1}}(1-E)-\omega_{s} \cdot\left(t_{1}-t_{0}\right)\right] \cdot \phi_{1}
$$

where $\epsilon_{1}$ is relative elastance during the first constant flow epoch from $t_{0}$ to $t_{1}$, and $\omega_{t 1}$ is the corresponding reciprocal time constant, according to Eq. 9. $E$ denotes an exponential given by $E=\exp \left\{-\omega_{t 1} \cdot\left(t_{1}-t_{0}\right)\right\}$.

Now, Eq. 8 is applied for another time, taking for $B_{e}(0)$, the value of $B_{e}\left(t_{1}\right)$ given by Eq. 12. Then, also

$$
B_{t}\left(t_{0}\right)=B_{t}\left(t_{1}\right)+\phi_{1} \cdot\left(t_{1}-t_{0}\right)
$$

This is substituted into Eq. 12, and the result together with, again, Eq. 7 into Eq. 8. This leads to an expression for $b_{e}(s)$ for the condition $t_{1}=0$. This expression is substituted into Eq. 10. After some straightforward algebra we obtain

$£\left[\frac{P(t)}{f(t)}\right]=e \cdot \frac{\epsilon_{1} \cdot \sigma}{\epsilon_{1}+\sigma} \cdot\left[\left\{1+\frac{\epsilon_{2}-\epsilon_{1}}{\epsilon_{1}} \cdot \frac{\omega_{s}}{s+\omega_{t 2}}\right\} \cdot\left(b_{t}-\frac{B_{d}}{s}\right)-\sigma \cdot \frac{\frac{1-E}{\omega_{t 1}} \phi_{1}+\frac{\phi_{2}}{s}}{\epsilon_{1} \cdot\left(s+\omega_{t 2}\right)}\right]$.

The time domain equivalent of Eq. 13 consists of four terms on the right hand side

$$
\frac{P(t)}{f(t)}=H_{1}(t)-H_{2}(t)-Z_{1}(t)-Z_{2}(t)
$$

The first term

$$
H_{1}(t)=e \cdot \frac{\epsilon_{1} \cdot \sigma}{\epsilon_{1}+\sigma} \cdot\left\{B_{t}(t)-B_{d}\right\}
$$

is the primary elastance term. According to Eq. 4, it equals isovolumic pressure at volume $b_{t}$. The second term is

$$
H_{2}(t)=e \cdot \frac{\epsilon_{1} \cdot \sigma}{\epsilon_{1}+\sigma} \cdot \frac{\epsilon_{1}-\epsilon_{2}}{\epsilon_{1}} \cdot \omega_{s} \cdot e^{-\omega_{t 2}\left(t-t_{2}\right)} *\left(b_{t}-\frac{B_{d}}{s}\right)
$$

where * denotes convolution over the time interval from $t_{1}$ to $t . H_{2}$ is unequal to zero only when $\epsilon_{2} \neq \epsilon_{1}$. As will be shown in results, this is the case for $\phi_{2}<\phi_{1}$. Equa- 


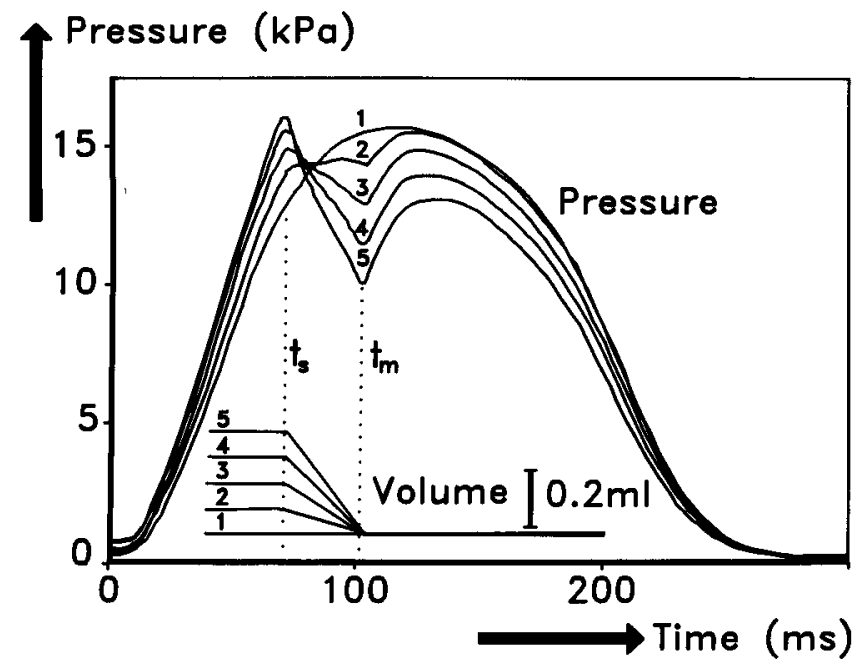

(a)

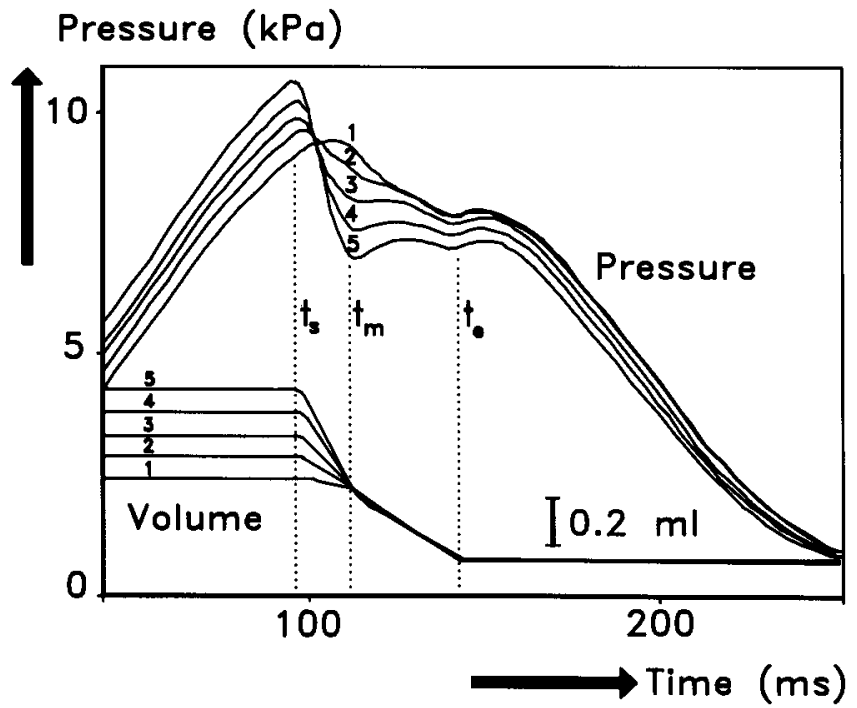

(b)

FIGURE 4. Pressure recordings during single and dual flow epochs. (a) Single flow epochs. From $t_{m}$ on for the five time courses shown, volume and time are equal, eliminating instantaneous effect of volume and time. Pressure curve 1 is an isovolumic recording: at $t_{m}$ resistance effect is zero. Time courses 2-5 show increasing constant flow values and increasing flow effects in the pressure tracings at $t_{m}$. After $t_{m}$ time courses 1-5 show a deficit with respect to isovolumic pressure, implying that pressure is deactivated by the stepwise flow decrease occurring at $t_{m}$. This deactivation relates uniquely and linearly to the resistance effect at $t_{m}$, irrespective of volume history. (b) Dual flow epochs. Time course 1: positive flow step at $t_{m}$. Time course 2: no flow step at $t_{m}$ (single flow epoch as a referencel. Time course 3, 4, 5: negative flow steps at $t_{m}$. From $t_{m}$ on, pressure tracings 1 and 2 are at the same pressure value, which is lower than isovolumic pressure. Since for pressure tracing 2 there is no deactivation, the equally lower value also for tracing 1 is due to the resistance effects only. Pressure tracings 3, 4, and 5 are progressively lower which can be ascribed to the negative, deactivating flow steps at $t_{m}$. Therefore, generally, at $t_{\theta}$, pressure is depressed as a combination of resistance and deactivation effects. In contrast, at $t_{m}$, pressure tracings are affected by resistance effects only. 
tion 16 , then, represents a low pass filtered step in elastance occurring at $t_{1}$. The filtering occurs with a cut-off frequency of $\omega_{i 2}$.

A single flow epoch can be represented by taking either $\phi_{1}$ or $\phi_{2}$ equal to zero. In the first case, (volume time course 1 in Fig. 3) Eq. 13 describes the pressure response during a single flow step. In the second case, corresponding to time course 6 , the equation represents the pressure response during the isovolumic period immediately after the single flow step. Then $b_{t}$ represents a constant volume: $b_{t}=B_{t} / s$. Accordingly, using Eq. 5

$$
\Delta \Pi_{d}=\frac{H_{2}(t)}{P_{\text {iso }}}=\frac{\epsilon_{1}-\epsilon_{2}}{\epsilon_{1}} \cdot \frac{\omega_{s}}{\omega_{t 2}} \cdot\left\{1-e^{-\omega_{t 2}\left(t-t_{1}\right)}\right\} .
$$

In Eq. $17 H_{2}(t)$ has been normalized with respect to isovolumic pressure, eliminating the influence of volume differences.

In the situation immediately following a constant flow epoch it is expected that $\epsilon_{2}<\epsilon_{1}$ (see results). Thus $\Delta \Pi_{d}$ is, then, positive and Eq. 17 indicates a pressure deficit in Eq. 14 to build up exponentially to a value equal to the coefficient: $\left[\left(\epsilon_{1}-\epsilon_{2}\right) / \epsilon_{1}\right] \cdot\left(\omega_{s} / \omega_{t 2}\right)$.

Normalizing the third term in Eq. 14 leads to

$$
\Delta \Pi_{f}=\frac{Z_{1}(t)}{P_{\text {iso }}}=\frac{\sigma}{\epsilon_{1}} \cdot \frac{1-E}{\omega_{t 1}\left(B_{t}-B_{d}\right)} \cdot e^{-\omega_{t 2}\left(t-t_{1}\right)} \cdot \phi_{1} .
$$

Thus, flow $\phi_{1}$ during the first constant flow epoch, after it has been switched to $\phi_{2}$ at $t_{1}$, elicits a switching transient during $t_{1} \rightarrow t_{2}$. This effect has an amplitude $\Delta \Pi\left(t_{1}\right)$ at $t_{1}$ equal to the coefficient of the exponential in Eq. 18. Because of the presence of $E$ in Eq. 18, first flow epochs (from $t_{0}$ to $t_{1}$ ), not long with respect to $1 / \omega_{t 1}$, will elicit smaller switching transients.

The combined effect $\Delta \Pi_{d}+\Delta \Pi_{f},\left(\phi_{2}=0\right)$, also is an exponential with a time constant $\omega_{t 2}$ decaying from a negative value equal to $\Delta \Pi_{f}$ at $t=t_{1}$ to the deactivation deficit $\Delta \Pi_{d}$ at $t \gg t_{1}$. Actually, Wijkstra and Boom $(109,110)$ showed the normalized pressure transient response following a constant flow in the isolated rabbit heart epoch to be exponential with an asymptotic pressure deficit.

The fourth term in Eq. 14 is given by

$$
Z_{2}=e \cdot \frac{\sigma^{2}}{\epsilon_{1}+\sigma} \cdot \frac{1}{\omega_{t 2}} \cdot\left\{1-e^{-\omega_{t 2}\left(t-t_{1}\right)}\right\} \cdot \phi_{2}
$$

showing how, during the second flow epoch, the flow effect of $\phi_{2}$ builds up to a steady value.

The exponentials in Eqs. 16, 18, and 19 are the result of redistribution of volume between the main and the series-elastic compartments caused by changes in pressure differences across the resistance. These, in turn, were elicited by the flow steps. The exponentials will be referred to as "flow switching effects."

Equation 13 will be validated experimentally, also as its normalized time domain equivalent $\Pi(t) / P_{\text {iso }}(t)$. The use of normalized pressure is advantageous since: First, it can be obtained experimentally if steady state isovolumic pressure is known. Secondly, its value is not influenced by effects introduced by the intrinsic time variability $f(t)$, and by differences in volume. 


\section{METHODS}

\section{Experimental Setup}

The effect of stepwise changes in ejection flow was studied in isolated rabbit hearts. Details of the physiological preparation, and the experimental set up have been described in detail elsewhere $(102,109)$. The hearts were quickly removed from the anesthetized animal (Hypnorm) and connected to a perfusion system, supplying oxygenated, Tyrode-like perfusion solution to the coronary arteries. For this, the arteries were cannulated by narrow catheters, which were glued to the cardiac tissue with tissue adhesive (Histoacryl blue). The aortic valves were glued shut in the same way.

The atria were removed from the heart and the ventricle was connected to a pump which was driven by a computer controlled, linear motor. The motor was powered by a high-power output amplifier, which received a fed-back position signal originating from a position transducer attached to the piston rod of the pump. The connection between ventricle and pump was made through a cannula sutured into the mitral orifice. The assembly consisting of ventricle, pump, linear motor, and power electronics had a volume step time of $2 \mathrm{~ms}$.

To stabilize the contraction frequency of the heart, it was stimulated artificially by supplying rectangular current pulses of 2-10 ms duration, and 2-10 A amplitude to two silver electrodes of $10 \times 10 \mathrm{~cm}$. Usually stimulation frequency was $1 \mathrm{~Hz}$. This low frequency was made possible by destroying the $\mathrm{A}-\mathrm{V}$ node, and thus, inducing a very low spontaneous contraction frequency of the ventricle.

Intraventricular pressure was measured with a catheter tip transducer (Honeywell/Philips Type 9815-156-1,000) positioned deep in the left ventricle and adjusted in such a way that "artifacts" resulting from Bernoulli effects $(53,75,76,77)$ were minimal. Pressure and position signals were sampled with a 12 bit A-D converter at $1 \mathrm{kHz}$.

Single and dual constant-flow epochs were imposed on the ventricle by corresponding constant velocity movements of the pump piston, according to Fig. 3. Flow patterns were either stepwise increasing or decreasing constant-flow epochs. Starting volumes

$$
B_{t}\left(t_{0}\right)=B_{t}\left(t_{m}\right)+\phi_{1} \cdot\left(t_{1}-t_{0}\right)
$$

epoch lengths $t_{m}-t_{s}$ and $t_{e}-t_{m}$, as well as flow intensities $\phi_{1}$ and $\phi_{2}$ during those epochs, could independently be varied, including the case that $\phi_{2}=0$. Within each protocol these variations were always realized in such a way that the volume time course during $\phi_{2}$ stayed unchanged. In this way, any differences in pressure response during $\phi_{2}$ were considered uncontaminated by influences of differing volume, time or flow, during the interval $t_{m} \rightarrow t_{e}$.

\section{System Identification}

Left ventricular pressure before, during, and after dual flow epochs was also computed by Eqs. 4 and 13. The computed pressures were then fitted to measured data as described previously (109). For the basic time function, $f(t)$, isovolumic pressure also measured, was utilized, and scaled to some suitable amplitude (see results). Using the SIMPLEX method according to Nelder and Mead (71) for the fitting procedure, 
iterations were continued until the difference between successive mean RMS errors of calculated pressure, with respect to measured pressure, was less than $10^{-10}$. The computations were performed on a time interval including the physiologic ejection period. Parameter values found by the fitting procedure were compared with values obtained by other methods.

\section{RESULTS}

\section{Ventricular Pressure During Single and Dual Flow Epochs}

Figure 4 shows representative examples of recorded single and dual-flow experiments as announced theoretically by Eq. 13 and Fig. 3. Pressure values of both single- and dual-flow epoch registrations are clearly affected by the changing volumes, and nonzero flow values during the flow intervals. As was explained in the Methods section, piston movements were arranged in such a way that from $t_{m}$ onwards, during the contraction cycle, volume time courses for different contraction cycles were identical. Hence, differences then observed in pressure time courses could be assumed uninfluenced by instantaneous volume, flow, and time differences.

For the single-flow epoch registration (Fig. 4a) the pressure-time course during $t_{s} \rightarrow t_{m}$ is described by Eq. 13 with $\phi_{1}=0, t_{1}=t_{s}$, and $t_{2}=t_{m}$, corresponding to volume time course 1 in Fig. 3. From $t_{m}$ onwards the same equation can be used with $\phi_{2}=0, t_{0}=t_{s}$, and $t_{1}=t_{m}$, volume time course 6 . During the $t_{s} \rightarrow t_{m}$ interval a pressure deficit develops. Pressure stays lower than isovolumic pressure values that would have been generated by the ventricle at volume $B\left(t_{m}\right)$. According to Eq. 13 there are at least two components expected to be superposed in the pressure deficit. First, there is an increasing elastance effect according to Eq. 15. The second component is a resistance effect expected to grow exponentially to a value given by Eq. 19.

At the end of a single constant ejection epoch $\left(t_{m}\right)$, these effects can be separated by examining constant-flow epochs with equal flow but different epoch durations as shown by Vaartjes and Boom (102), (not shown here). This was done by applying Eq. 13 with $\phi_{2}=0, t_{0}=t_{s}, t_{1}=t_{m}$, and $\epsilon_{1}=1$, varying $E$ by varying $t_{m}-t_{s}$. Thus, for the isolated rabbit heart at $37^{\circ} \mathrm{C}$, a value for the time constant $1 / \omega_{t 1}$ could be established of $10.35 \pm 0.26 \mathrm{~ms}$.

After the single flow epoch, immediately following $t_{m}$ (Fig. 4), according to Eq. 13, there is no resistance effect from $\phi_{2}$ expected: $Z_{2}=0$ since $\phi_{2}=0$. Wijkstra and Boom (109) showed that this part of the pressure response, after normalizing by dividing the signal by isovolumic pressure, is exponential with an asymptotic value staying below 1. This was interpreted as the effects of $\Delta \Pi_{d}+\Delta \Pi_{f}$ from Eqs. 17 and 18, respectively. Indeed, the time constant was found equal to $1 / \omega_{i 2}=10.44 \pm 0.09 \mathrm{~ms}$, in good agreement with the above mentioned value for $1 / \omega_{t 1}$. Thus, redevelopment of normalized pressure $\Pi(t)=P(t) / P_{\text {iso }}(t)$ was found to be incomplete as predicted theoretically by Eq. 13. The result agreed quantitatively with Eq. 13 with a nonzero $H_{2}$ term. Thus, $\epsilon_{2}<\epsilon_{1}$, and it could be concluded that in the isovolumic phase, following a constant ejection flow epoch, a depression of elastance occurs. In accordance with prevailing literature (for instance Hunter [48]), this depression will be referred to as "deactivation."

According to Wijkstra and Boom (109), the single flow epoch deactivation after $t_{m}$ is related uniquely and linearly to the resistance effect that was developed at $t_{m}$, that is, the value of $\Delta \Pi_{f}$ in Eq. 18 for $t=t_{1}$. This equation predicts $\Delta \Pi_{f}\left(t=t_{1}\right)$ to 
depend on flow intensity $\left(\phi_{1}\right)$ as well as, for the shorter flow epochs, on duration of the interval $t_{s} \rightarrow t_{m}$ (because of the flow-switching effect, represented by $E$ ). It was observed that deactivation did not depend on flow intensity $\left(\phi_{1}\right)$ or flow duration $(E)$ separately, but only their combined influence in Eq. 13 at $t=t_{1}$.

In Fig. 4a the deficit, growing for increasing values of $\phi_{1},\left(\phi_{2}=0\right)$, in isovolumic pressure after $t_{m}$ is clearly visible. The selective dependence of deactivation on the resistance effect at $t_{m}\left(=t_{1}\right)$, allowed the conclusion that there was an intimate link of deactivation with the conditions existing at the negative flow step at $t_{m}$, and that no further influence of the flow and volume history of the constant-flow epoch existed.

Thus, negative flow steps, like those occurring at $t_{m}$ (Fig. $4 \mathrm{a}$ ) induce deactivation. The analysis was based on the predictive validity of Eq. 13 with $\epsilon_{1}=1$, both during the interval $t_{s} \rightarrow t_{m}$ as well as during the isovolumic contraction phase before $t_{s}$. This implies, that following the positive flow step at $t_{s}$, there was no deactivation.

Generalizing, we now hypothesize that following any positive flow step, starting at any flow value, deactivation fails to occur. As a corollary, we have to assume that deactivation occurs as a result of negative flow steps.

Turning to Fig. $4 \mathrm{~b}$, the depression of pressure at $t_{e}$, at the end of a dual constant flow epoch, is thus, not necessarily a mere resistance effect for all flow interventions involved. This is in contrast to the situation at $t_{m}$ in both Fig. $4 \mathrm{a}$ and Fig. $4 \mathrm{~b}$. At $t_{e}$ two flow steps have occurred, one positive at $t_{s}$, similar to that in Fig. $4 \mathrm{a}$, and one that was either positive/zero, or negative. Figure $4 b$ shows that for the one increasing step at $t_{m}$ as well as for the nonstepping flow epoch (which is identical to a single-flow epoch), pressure depressions both at $t_{m}$ and $t_{e}$ equal. This is in accordance with the assumption that for those two experiments pressure depressions at $t_{e}$ do constitute a pure resistance effect. In contrast, the two decreasing flow steps at $t_{m}$ elicit greater pressure deficits at $t_{e}$. Those negative flow steps at $t_{m}$ are therefore associated with deactivation observable at $t_{e}$.

A difference between negative flow steps occurring at either $t_{m}$ and $t_{e}$ is that at the latter instant flow steps to zero, leaving the value of $\phi_{2}$ as the only degree of experimental freedom. A possible relationship between deactivation after $t_{e}$, and the flow effect at $t_{e}$ could, therefore, be straightforwardly established for deactivation following single flow epochs, in the same way as it was done at $t_{m}$ by Wijkstra and Boom (109). In contrast, in Fig. 4b, at $t_{m}$ flow steps from one nonzero value to another, so that two parameters are involved, both $\phi_{1}$ and $\phi_{2}$. Consequently, the ensuing depression of pressure cannot be expected to be as simply related to the previous flow effect.

Wijkstra and Boom (109) showed that deactivation brought about by stepping to zero-flow stayed unchanged during the remaining systolic phase. Also, the amount of deactivation elicited by a given amount of flow effect, if imposed at different times during systole, was independent of that time. We will assume that this constancy also holds for the effect of nonzero stepping flow during the interval $t_{m} \rightarrow t_{s}$, while flow is $\phi_{2}$. We will also assume that the additional pressure deficit resulting from the negative flow stepping at $t_{m}$ can be interpreted as deactivation, that is: a decrease of elastance, as it was elicited at $t_{m}$. The amount of deactivation may be related to the flow step parameters at $t_{m}$. For this, again, normalized pressure $\Pi(t)=P(t) / P_{\text {iso }}$ will be calculated by dividing relevant pressure values by corresponding values of the isovolumic pressure signal for the same volume. 


\section{Depression of Elastance After a Stepped Flow Epoch}

After a constant-flow, epoch redevelopment of normalized pressure $\Pi(t)$ is exponential (109) with time constant $1 / \omega_{t 1}$, and a deactivated value of $\epsilon$. For single constant-flow pulses, the amount of deactivation following the zero-flow step at $t_{m}$ could be identified in this way.

This procedure can be followed in principle whenever redevelopment of pressure $P(t)$ occurs isovolumically. Then $B_{t}$ is constant: $B_{t}=s \cdot b_{t}$, and the convolution in Eq. 16 reduces to a simple multiplication. This is the case after the dual-flow pulse response of Fig. 4b. The quality of an exponential fit of the isovolumic phase following $t_{e}$ is shown in Fig. 5. It also demonstrates that in the case of a dual flow pulse history redevelopment of $\Pi(t)$, values are exponential.

The asymptotic value of $\Pi(t)$ appears to be well defined since the quality of the fit is high. Similarly to the single flow epoch situation, at the end of the exponential, a plateau is reached of normalized pressure. This can thus be associated with a state of permanently depressed elastance. Figure 6 shows that the level of the plateau values as a function of normalized pressure at $t_{e}$. These are the values, which for single-flow responses would correspond to the resistance effect. However, for step-wise decreasing dual-flow responses, after $t_{e}$ are contaminated with deactivation, elicited at $t_{m}$ by the negative flow step occurring there.

From Fig. 6 three conclusions were drawn. First, the relation between deactivation, and end-ejection normalized pressure is linear and independent of the flow-step parameters. Second, positive flow steps at $t_{m}$ elicit a deactivation at $t_{e}$ independent of their flow step parameters and equal to the deactivation found for a single-flow epoch. Clearly, as discussed above, the deactivation that is brought about in that case results from the negative flow step to zero at $t_{e}$ only. The third conclusion is drawn from the fact that each of the two protocols shown in Fig. 6 contains one beat with equal flow before and after the step at $t_{m}$. Accordingly, the relation between deactivation and the end-systolic resistance effect, as represented by $\Pi(t)$ being unequal to 1 , is equal for constant-flow epochs and flow-switching epochs. This strongly suggests, that after either a step, or a constant-flow, elastance is deactivated according to a similar mechanism.

The relation depicted by Fig. 6 is not sufficient to calculate ventricular pressure for any (discretized) variable flow. For this, deactivation during changing flow, not only at the end of it, has to be examined.

\section{Depression of Elastance Caused by a Flow Step During Ejection}

For assessing deactivation during the interval $t_{m} \rightarrow t_{e}$, the method of exponential fitting cannot be applied since volume then is changing, and Eq. 16 does not predict an exponential. The full convolution would then have to be dealt with. However, as stated above, it could be assumed that deactivation induced by the negative flow step at $t_{m}$ lasted throughout the second constant flow phase and was constant. Figure 7 shows normalized pressure at $t_{e}$ as a function of the flow of the first flow phase $\phi_{1}$. For values of $\phi_{1}>\phi_{2}$, thus for those $\phi_{1}$ corresponding with a negative flow step at $t_{m}$, normalized pressure at $t_{e}$, or $\Pi\left(t_{e}\right)$, decreases for increasing step size. The decrease is stronger if the interval $t_{s} \rightarrow t_{m}$ is longer, causing the resistance effect at $t_{m}$ to be greater. (In the case of Fig. 7, the length of $t_{s} \rightarrow t_{m}$ was not large with respect 


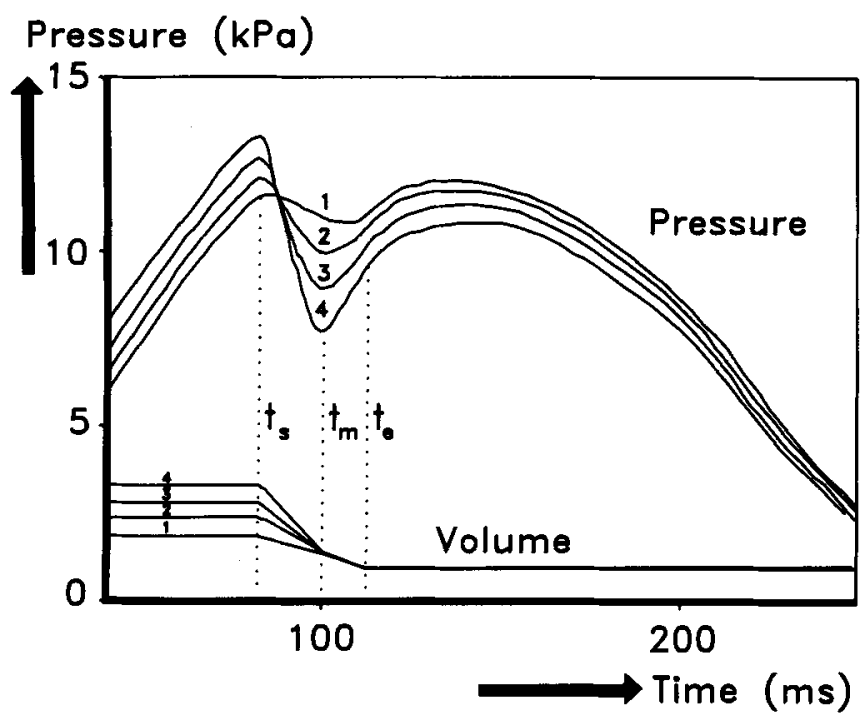

(a)

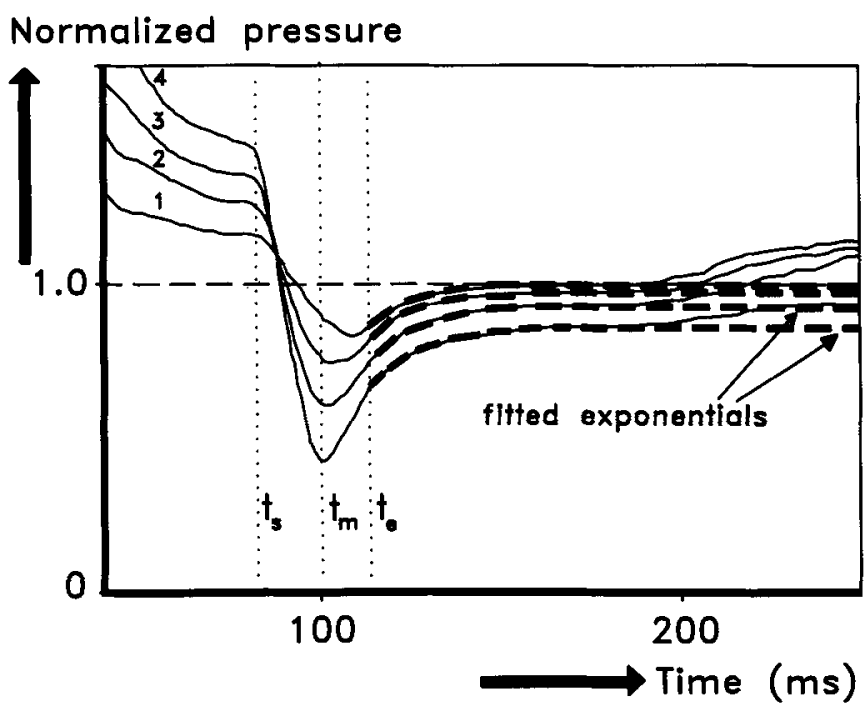

(b)

FIGURE 5. Redevelopment of pressure after a dual flow step. (a) Pressure and volume tracings with negative flow steps at $t_{m}$. (b) Normalized pressure calculated by dividing actual measured pressure values by corresponding isovolumic pressure values, as a function of time during and following dual constant-flow epochs. The isovolumic recovery phase following $t_{m}$ was fitted by an exponential which made the determination of a plateau value possible. This plateau was assumed to reflect deactivation. Nondeactivated values would correspond to a plateau value $=1$. For the exponential regression analysis all sampled points between the start of the exponential $\left(t_{m}\right)$ and the end of the plateau (estimated "by eye") were used. The end of the plateau was found to be at $193 \pm 25 \mathrm{~ms}$ (mean \pm SD), which is well after the end of the physiological ejection period. The total averaged RMS difference between fitted exponential and measured normalized pressure values was calculated to be $0.0077 \pm 0.0036$ (mean $\pm S D$ ), which has to be compared to the nondeactivated value, which $=1$. This indicates excellent fit and allows the conclusion that normalized pressure recovers exponentially from a dual-flow epoch, and that deactivation established in this way is well defined. 


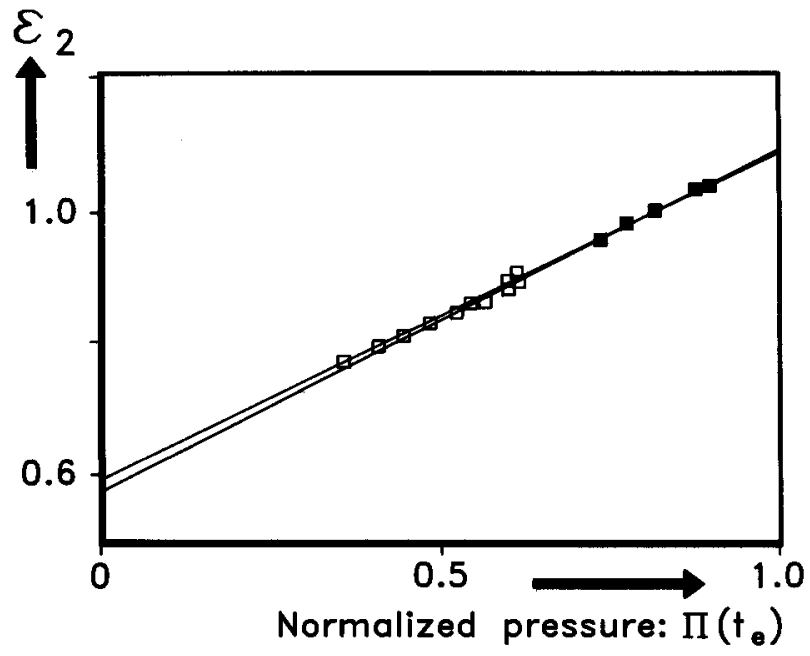

FIGURE 6. Deactivation represented by the parameter $\epsilon_{2}$ during the interval $t_{m} \rightarrow t_{\theta}$ (Fig. 5) as a function of normalized pressure at $t_{m}$. Filled squares: $\phi_{2}=10 \mathrm{ml} / \mathrm{s}, t_{\theta}-t_{m}=12 \mathrm{~ms}$. Open squares: $\phi_{2}=10 \mathrm{ml} / \mathrm{s}, t_{\theta}-t_{m}=31 \mathrm{~ms}$. The different parameters for $\phi_{2}$ result in different resistance effects at $t_{m}$, reflected by different normalized pressure values II. Despite this, $\epsilon_{2}$ values have the same relationships to normalized pressure. In both protocols one flow epoch does not step, and thus is a single flow epoch. It falls on the same relation. Therefore, after both dual and single flow epochs, the relation between deactivation and normalized pressure is unique.

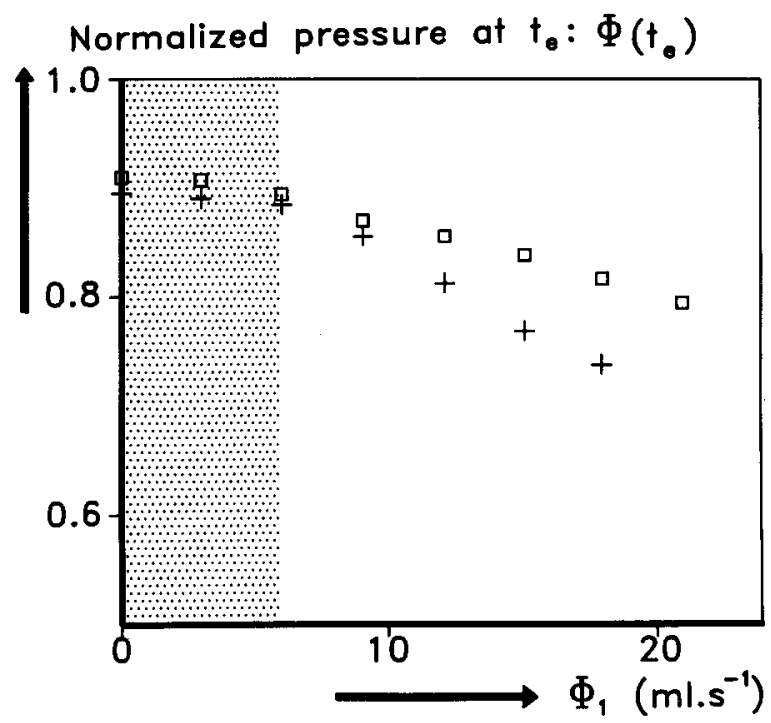

FIGURE 7. Deactivation at $t_{\theta}$ caused by flow steps at $t_{m}$. Normalized pressure at $t_{\theta}$, representing both the resistance effect and possible deactivation resulting from a (negative) flow step at $t_{m}$; as a function of the value of the flow of the first flow phase of a dual flow epoch. Shaded area corresponds to increasing or zero flow steps. For negative flow steps, normalized pressure decreases for increasing flow step magnitude. Since the second flow value is equal for all steps, resistance effects are equal and the difference in normalized pressure at $t_{e}$ is due to different values of deactivation. Open squares: $t_{m}-t_{s}=15 \mathrm{~ms}$. Crosses: $t_{m}-t_{s}=22 \mathrm{~ms}, t_{e}-t_{m}=30 \mathrm{~ms}, \phi_{2}=6 \mathrm{ml} / \mathrm{s}$. 


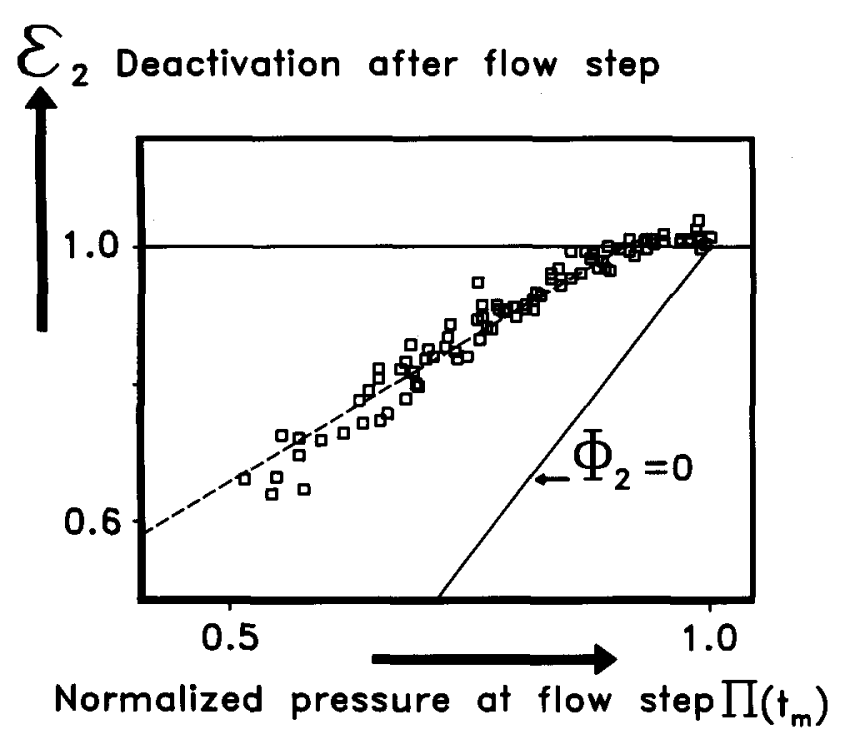

FIGURE 8. Deactivation elicited by step wise changes of ejection flow as a function of normalized pressure. On the vertical axis the resistance effect measured during 12 protocols from 5 experiments at $t_{m}$ (see Fig. $4 b$ ) is plotted. The pressure depressions at $t_{\theta}$ were corrected for the resistance effect occurring for positive flow steps. Positive flow steps were shown not to elicit deactivation in this study and thus to exhibit a pure resistance effect only. All dual flow epochs had equal second flow phases regarding volume, flow and time, and, therefore, also had equal resistance effect (see Fig. 2). The resulting deactivation expressed by the value of $\epsilon$ at $t_{\theta}$ was assumed to be equal to deactivation at $t_{m}$ (see text). Deactivation is shown to be linearly dependent on normalized pressure regardless of values of absolute pressure, $\phi_{1}, t_{m}-t_{s}$, or the ventricle under study. Drawn line: best fitting straight line "by eye." Broken line: the same relation for $\phi_{2}=0$, according to Fig. 7 .

to the time constant $1 / \omega_{t}$ of the flow switching effect at $t_{s}$, causing the factor containing the exponential in Eq. 19 to be nonzero.) In contrast, both positive and zeroflow steps at $t_{m}$ result in a pressure decrease at $t_{e}$ of the equal magnitude. As was reasoned earlier, this represents the resistance effect caused by flow $\phi_{2}$ only.

Since the resistance effect at $t_{e}$ is different for different $\phi_{1}$ values and also for different ventricles, plots like Fig. 7 are difficult to compare for different situations. Therefore, data as shown in Fig. 7, were replotted so as to compensate for the resistance effect given by the coefficient of the exponent in Eq. 19. For the experiments of Fig. 7, generally, $t-t_{1} \gg 1 / \omega_{t 2}$.) We are looking for a relation between elastance depression at $t_{m}$ and ejection parameters at that same instant. Since up to this point deactivation depended uniquely on normalized pressure at $t_{m}$, this quantity was tried as the independent variable also in the present case. Figure 8 shows the result. Clearly, for determining the amount of deactivation at $t_{e}$, and thus at $t_{m}$ as well, for the $\phi_{2}$ value involved, $\Pi\left(t_{m}\right)$ is the essential determining variable. Again, as at $t_{e}$, elastance depression is determined by the value of normalized pressure at the time the depression is elicited. It is not influenced by either $\phi_{1}$ or the length of the interval $t_{s} \rightarrow t_{m}$ separately. Only their combined influences on $\Pi\left(t_{m}\right)$ as expressed by the coefficient of the exponential in Eq. 18 appear to be relevant. The relation between deactivation 
and normalized pressure is linear for those values of $\Pi\left(t_{m}\right)$ that correspond to negative flow steps at $t_{m}$.

The relation shown in Fig. 8 can be represented algebraically by

$$
\begin{array}{ll}
\epsilon=\alpha \Pi \quad\left(\phi_{1}>\phi_{2}\right) \\
\epsilon=1 \quad\left(\phi_{1} \leq \phi_{2}\right) .
\end{array}
$$

In (20) $\alpha$ is the slope of the linear part of the relation shown in Fig. 8 . It is dependent on $\epsilon$ as well as on the horizontal offset in Fig. 8. The offset is not equal to 1 since, for all positive flow steps at $t_{1}, \epsilon=1$. The range of this is dependent on $\phi_{2}$, since for the largest value of $\phi_{1}$, for which there is no deactivation, it holds that $\phi_{1}=\phi_{2}$.

\section{System Identification}

The pressure generated by the left ventricle was simulated using the three-compartment model introduced in the Theory Section by Eqs. 4-22. Following Wijkstra and Boom (110), the time function $f(t)$ was derived from measured isovolumic pressure by

$$
f(t)=\frac{P_{\text {iso }}}{B_{t}-B_{d}}
$$

Implying that

$$
\frac{\sigma \cdot \epsilon_{1}}{\sigma+\epsilon_{1}} e=1
$$

taking for $B_{t}$ the volume at $t_{e}$, and for $\epsilon_{1}$ undeactivated elastance before $t_{m}: \epsilon_{1}=1$. Flow $\phi_{1}$ was taken as the experimental variable. The computation was started with educated guesses for $B_{t}-B_{d}, \sigma, \mu$, and $\epsilon_{2}$, deactivated elastance between $t_{m}$ and $t_{e}$. Elastic volume $B_{e}$ was calculated for the first constant flow phase of a dual flow epoch. At the moment of the flow step $\left(t_{m}\right), \epsilon_{1}$ was replaced by a tentative value $\epsilon_{2}$, and the computation of $B_{e}$ was continued. Thus, $B_{e}(0)$ for the second constant-flow phase was equal to the value of $B_{e}$ calculated at the end of the first.

Ventricular pressure until $t_{e}$ was computed by the time-domain equivalent of Eq. 13 . Following $t_{e}$, a third deactivation elastance was computed from $\epsilon_{3}=a \cdot \mathbf{\Pi}\left(t_{e}\right)+b$, and the computation of pressure was continued until $200 \mathrm{~ms}$ after stimulation. The RMS deviation of the calculated $P(t)$ from the experimental was computed and the process was iterated until minimal RMS error. In order to test the sensitivity of the model for the presence of deactivation in the model, two more cases were investigated in this way. In one case, elastance was assumed constant, both during flow stepping, and at the end of the dual-flow pulse: $\epsilon_{3}=\epsilon_{2}=1$. In a second case, deactivation was assumed to be elicited at the end of the dual-flow pulse only. A representative example of the comparison of the experimental, and the fitted result is shown in Fig. 9. The estimated values of the model parameters are given in Table 1. Figure 9 shows that the three-compartment model gives satisfactory fitting results, be it only when, at all negative flow steps, deactivation is assumed to occur. Table 1 shows that RMS 


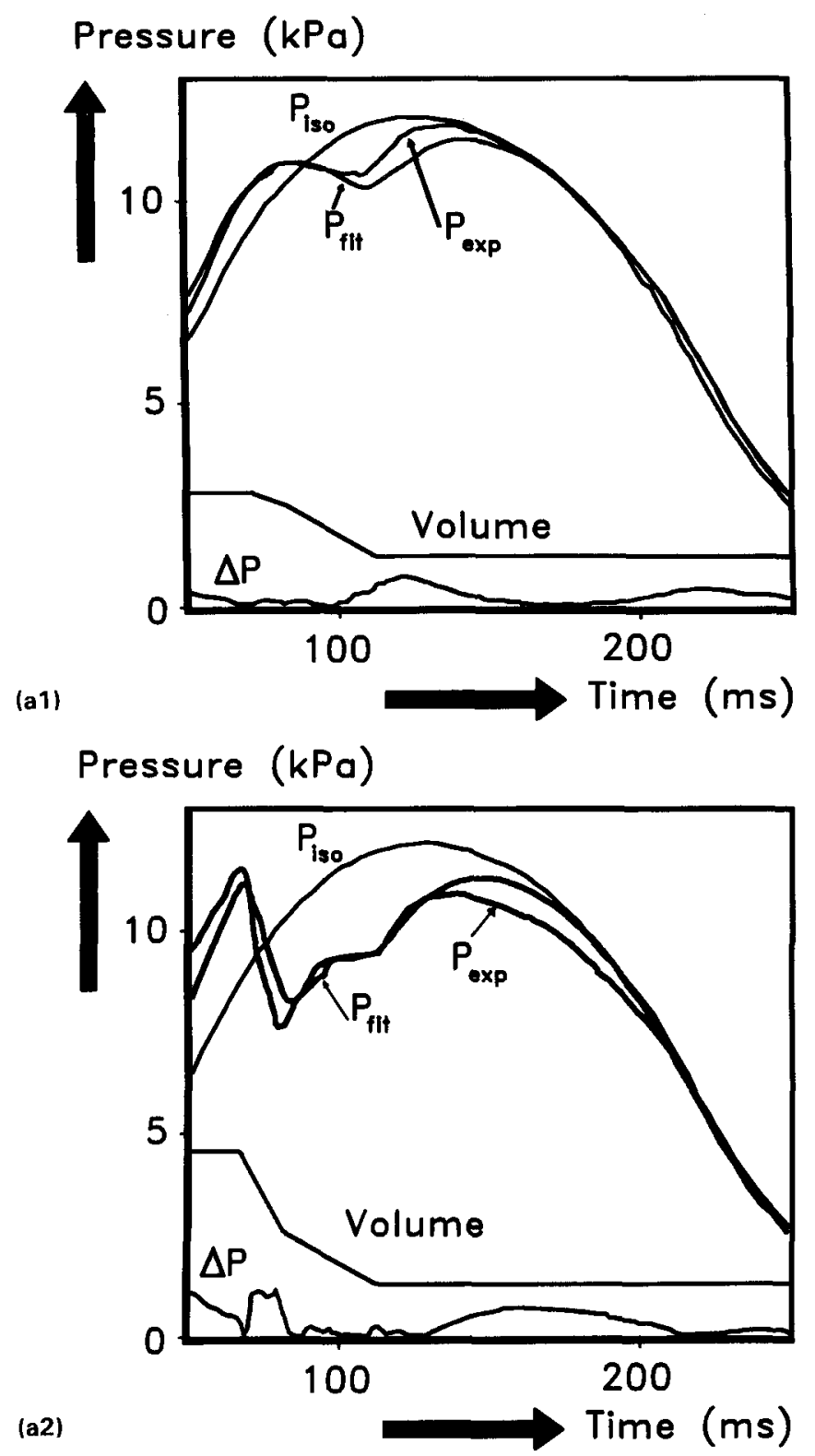

FIGURE 9. Fitting of the three-compartment model on experimental dual-flow epochs. All panels: $\phi_{2}=6 \mathrm{ml} / \mathrm{s}, t_{\theta}-t_{m}=6 \mathrm{ml} / \mathrm{s}$. The fitting was performed in the interval 55-200 ms after stimulation of the ventricle. $\boldsymbol{P}_{\text {iso }}$ : isovolumic pressure; $\boldsymbol{P}_{\text {fit }}$ : simulated pressure; $\boldsymbol{P}_{\text {exp }}$ : measured pressure; $\Delta P$ : absolute difference between simulated and experimental pressure. (a1), (b1), (c1): $\phi_{1}=3 \mathrm{ml} / \mathrm{s}, t_{m}-$ $t_{s}=15 \mathrm{~ms}$. (a2), (b2), (c2): $\phi_{1}=21 \mathrm{ml} / \mathrm{s}, t_{m}-t_{s}=15 \mathrm{~ms}$. (a1), (a2): model without deactivation. (Figure continued on following two pages.)

errors are higher if such deactivation is not incorporated. Also, parameter values found for that case are different from those found with the complete model. For instance, series-elastance would be underestimated without deactivation while resistance would be overestimated. 

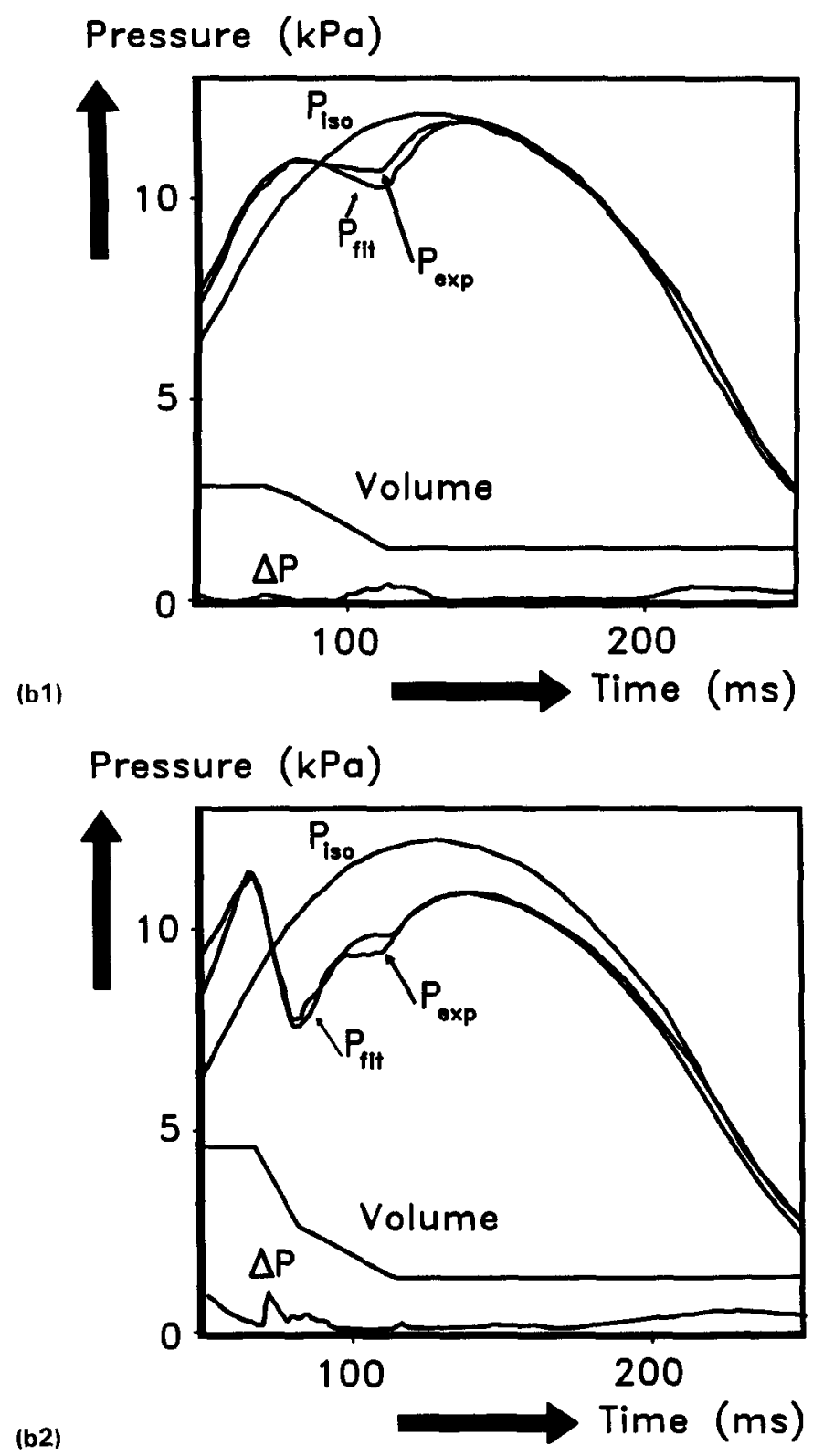

FIGURE 9 continued. (b1), (b2): model with activation elicited at end systole only.

\section{DISCUSSION}

Contrary to that proposed in the present study, global models of the left ventricle containing elastances and resistance only, are not capable to accurately describe ventricular pressure generation. Campbell et al. (22) examined a number of such models and concluded that all had shortcomings during the second half of the ejection phase. The results of the present study explain this finding. The physiologic flow time course has a triangular shape. During the first third of ejection, flow is increasing to 


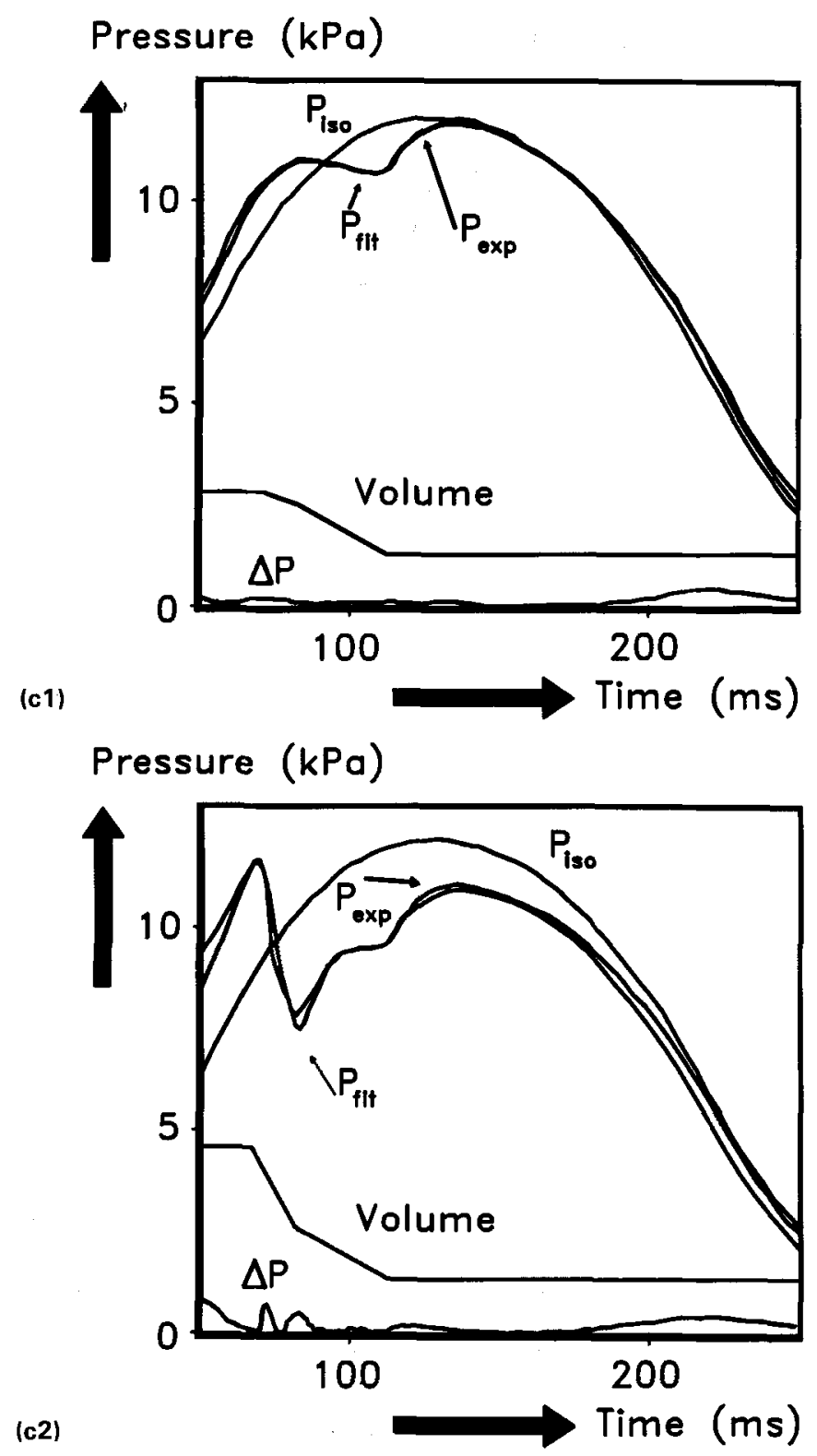

FIGURE 9 continued. (c1), (c2): model with activation elicited both at end-systole $\left(t_{\theta}\right)$ and at the time of the flow step $\left(t_{m}\right)$. A good fit is obtained with (c1) and (c2) only, demonstrating the necessity of deactivation in the model as well as its correctness.

a crest value (88). No deactivation is expected during this ascending phase and elastance-resistance models can be adequate. In contrast, in the second two thirds of systole, flow decreases, and according to the results of the present study, elastance depression will manifest itself. This phenomenon is not accounted for by pure 
TABLE 1. Parameter values of the model found after fitting two dual-flow epochs with different flow parameters to experimental results.

\begin{tabular}{|c|c|c|c|c|c|c|c|c|}
\hline \multirow[b]{3}{*}{ Condition } & \multicolumn{8}{|c|}{ Parameter } \\
\hline & \multirow{2}{*}{$\begin{array}{c}B_{t}-B_{d} \\
(\mathrm{ml})\end{array}$} & \multirow[b]{2}{*}{$e$} & \multirow{2}{*}{$\begin{array}{c}\sigma \\
(s)\end{array}$} & \multirow[b]{2}{*}{$\mu$} & \multicolumn{2}{|c|}{$\epsilon_{3}$} & \multirow[b]{2}{*}{$\epsilon_{2}$} & \multirow{2}{*}{$\begin{array}{c}\text { RMS } \\
\text { error } \\
(\mathrm{mmHg})\end{array}$} \\
\hline & & & & & $a$ & $b$ & & \\
\hline$\epsilon=1$ & 1.76 & 1.40 & 2.48 & 0.075 & 0 & 1 & 1 & 3.19 \\
\hline$\epsilon<1$ after $t_{e}$ & 1.53 & 1.27 & 3.73 & 0.051 & 0.83 & 0.26 & 1 & 1.71 \\
\hline$\epsilon<1$ after $t_{m}$ & 1.48 & 1.17 & 5.8 & 0.035 & 0.77 & 0.30 & 0.91 & 1.24 \\
\hline
\end{tabular}

Symbols used: $B_{t}-B_{d}, e, \sigma, \mu$ (see text); $\epsilon_{3}$ : deactivation factor after the end of ejection. $a$ and $b$ are the parameters in: $\epsilon_{3}=a \cdot \Pi\left(t_{e}\right)+b$. First row: no deactivation; second row: deactivation after $t_{e}$; third row: deactivation after both $t_{e}$ and $t_{m}$. In the third row the RMS error is minimal, indicating optimal fit. Estimated active volume $B_{t}-B_{d}$ has a realistic value for the rabbit ventricle. Leaving out deactivation from the model underestimates series-elastance and overestimates resistance in the ventricle.

elastance-resistance models. On the cellular (59), as well as on the muscular $(16,18$, 33,73 ) level, it has long been known that the capability to redevelop force after fiber shortening, is negatively affected. The present study, however, indicates that it is the shortening itself, rather than the state of having shortened that is associated with deactivation.

Others have found that elastance amplitude in the intact ventricle is not constant during systole. A number of ejection parameters were claimed to influence the time course of elastance. Increasing stroke volume and/or ejection fraction $(8,97)$, as well as the preload to the ventricle $(8,28)$, were shown to result in a decrease of elastance. In contrast, after increasing afterload, the opposite occurred $(8,31,84)$. These findings were confusing since, for instance, preload, either to heart muscle $(38,44)$, or to the intact ventricle $(61,84)$, as well as afterload $(84)$, were found to enhance the contractile state. The results of the present study suggest how at least parts of this seeming contradiction can be resolved. Deactivation is associated with flow decrease, to an extent closely linked to the level of the flow effect measurable at the instant that flow actually changes. Interventions that result in higher flow will also result in a greater flow effect (consisting partly of resistance and partly of deactivation accumulated as a result of earlier flow decreases). A higher flow also implies a more pronounced flow decrease in the latter phase of ejection. This stronger decrease, together with the greater flow effect, yield a greater deactivation. Afterload or preload increases results in smaller or greater ejection flow, respectively.

The conclusion of the present paper could not have been drawn by the above authors, since they applied physiological flow patterns, in an almost self-evident approach. The study of the present paper, showing that it is flow decrease that is necessary to make deactivation manifest, was based on the reasoning that if flow is expected to have influence on elastance, flow epochs consisting of one or more constant flow phases, would reveal the dependence more readily. This reasoning is analogous to the approach in the past, when it was felt that since ventricular volume 
probably was the prime determinant of pressure, studying the ventricle under constant volume conditions would be illuminating $(39,82)$. As it turned out, a constant flow epoch is the only flow pattern eliciting elastance depression at one instant only; the very end.

Whereas, it is clear that the decreased capability of the ventricle to regenerate pressure after a negative flow step is compatible with a change in elastance, it might be questioned to what extent parameters other than elastance are also in play. Within the scope of the global three-compartment model posed in the present study, one or more of the parameters primary elastance, resistance, or series elastance are candidates for change after a negative flow step. If none of them changed, this would be a reason for rejecting the model. During the isovolumic recovery phase, after a constant-flow step, normalized pressure accurately follows an exponential time course $(109,110)$. Its time constant did not significantly differ from the time constant measured for the switching-on effect observed directly following the positive flow step at the beginning of the constant-flow epoch. This latter, positive flow step does not induce deactivation. The time constant, according to the model is given by

$$
\tau=\frac{1}{\omega_{t}}=\frac{\mu}{\sigma+\epsilon}
$$

Series-elastance $\sigma$ is appreciably greater than depressed elastance $\epsilon$. Therefore, the only two possibilities which do not affect $\tau$ appreciably are: a change in both $\mu$ and $\sigma$ keeping their ratio approximately constant, or a change of $\epsilon$. The simulations were done to check this point, among others. It was shown that a satisfactory model fit resulted when assuming that $\mu$ was not changed by the negative flow steps at $t_{m}$. If $\mu$ is not changed, $\sigma$ cannot be changed without changing the time constant. It is therefore concluded that also during flow, a negative flow step results in a depression of elastance $\epsilon$.

A global model such as that proposed in the present study gives no direct clues as to the physiological significance of its structure and its parameters. A primary aim of global models of the ventricle is to serve as a tool for the definition and the experimental derivation of ventricular performance indices that may be of clinical or physiological significance. For instance, the way in which the ventricle matches its performance to the properties of the arterial tree can be expressed in terms of a global model (106). Nevertheless, the response of the ventricle to step-wise flow changes is so conspicuously transparent (see for instance Fig. 8), that it is tempting to interpret it in terms of physiologic functioning of myocardial muscle. The way to do so would be through the construction of a detailed ventricular model, based on actin-myosin filament interaction $(36,42,49,56,78)$, and ventricular geometry $(17,30,41,46,54,65,66$, $79,81)$, as well as anatomy $(4,81,90,92,104)$, and electrophysiology $(10)$. However, such studies seldom attempt to quantitatively predict ventricular performance in terms of compressed data models as the present three-compartment model. Usually, a forcevelocity relation for heart muscle is built in $(6,10,17)$, but then it is not explained how ventricular pressure-flow relations are linear $(87,102)$, whereas force-velocity relations for cardiac muscle are hyperbolic $(24,89)$. One reason for the difference might be that ventricular geometry, both with respect to muscle fiber orientation and complexity 
of shape, has not been accounted for in sufficient detail. Without a global model available this discrepancy would not have been detected.

The same holds true for deactivation of elastance in the ventricle. This phenomenon plausibly corresponds with a depressed force-length relation in cardiac muscle $(18,33)$. It is interesting to note that Edman and Nilsson (33) found that in cardiac muscle, after constant velocity shortening contraction, force is decreased to an amount related to the shortening velocity. However, in the simpler geometrical models, the relationship between shortening velocity and ejection flow is not linear, so that the transformation is quantitatively not self-evident.

The remarkable link between pressure depression during flow and the inability of the ventricle to regenerate full isovolumic pressure, suggests that the two phenomena are an expression of the same myocardial mechanism. Also, here a simple translation to myocardial events is difficult. Force and pressure are thought to be generated by discrete molecular force generators, the number of which determines the amount of force, or pressure (34). Shortening of muscle fibers is thought to couple generators to an amount closely linked to shortening velocity, a mechanism that can quantitatively explain the force-velocity relation $(9,35,51)$. Elastance depression, then, corresponds to the property that the muscle is not capable of regenerating the same amount of force generators as before decoupling. It is not implausible that the more force generators were decoupled, the more recouplable generators were lost.

One way to account for the link between flow effect and elastance depression consists in the assumption that it is decoupling itself that introduces the depression of elastance. Each force generator that is decoupled, then, would naturally cause a fixed amount of depression of regeneration capability. This view implies that elastance depression occurs during flow instead of after the end of it. Moreover, decoupling of force generators is a reversible process. More generators are decoupled than, at the end, correspond with the number of generators left, since during flow a number are recoupled as well $(35,51)$. The implication would be that part of resistance, as measured during the present study, is identical with elastance depression after the end of ejection. However, no definitive conclusions can be drawn on the basis of the material presented in the present study. It may also be questioned whether this distinction would be observable in a global model of the ventricle.

The results summed up in Fig. 8 now make it possible to propose a complete state model of the generation of left ventricular pressure for arbitrary, including physiologic flow patterns. Such a model should take into account the observation reported on in the present paper. Of these, the dependence of deactivation, $\epsilon$, on instantaneous normalized pressure, $\Pi(t)$, (or flow effect, which is equivalent) is the most essential. This relationship is illustrated by Fig. 8, and expressed by Eq. 20. However, this complicates Eq. 6 because therein $\epsilon$ was introduced as a constant. If we admit, nevertheless, $\epsilon$ to be variable, while requiring that Eq. 6 retains its validity, this would change Eq. 6 into an alinear differential equation.

Furthermore the findings of Eq. 20 have to be expanded since, in the present study, only one nonzero $\phi_{2}$ value was studied extensively. However, Fig. 4 shows that for the case $\phi_{2}=0$ (isovolumic recovery condition) the relation between elastance depression $\epsilon$ and $\Pi$ is steeper than for $\phi_{2}$ not equal to zero. This makes an estimation of $d \epsilon / d \phi \simeq \Delta \epsilon / \Delta \phi$ possible. The estimated values of $\Delta \epsilon$ and $\Delta \phi$ are indicated in Fig. 8. 
Thus

$$
\frac{d \epsilon}{d t}=\frac{d \epsilon}{d \phi} \cdot \frac{d \phi}{d t} \simeq \frac{\Delta \epsilon}{\Delta \phi} \cdot \frac{d \phi}{d t}
$$

and Eq. 20 for negative values of $d \phi / d t$ :

$$
\begin{array}{lll}
\frac{d \epsilon}{d t}=\left[\beta \cdot \Pi+\alpha \cdot \frac{d \Pi}{d \phi}\right] \cdot \frac{d \phi}{d t}, & \frac{d \phi}{d t}<0 \\
\frac{d \epsilon}{d t}=0 & \frac{d \phi}{d t} \geq 0
\end{array}
$$

were $\beta=d \alpha / d \phi$.

In Fig. 8 the case $\phi_{2}=0$ is also indicated. It shows that $\mathrm{d} \epsilon / d \phi$ depends much more on variations in $\alpha$ than on those in $\Pi$. Therefore the second term on the right in Eq. 25 will be neglected.

Although the value of $\beta=d \alpha / d \phi$, thus, could be estimated from Figs. 6 and 8 , it is more realistic to consider it as an additional parameter to be estimated from measurements. Also, as yet, there are no further data available on how this parameter, that is expected to be closely linked to the contractile machinery of the cardiac muscle in various mammalian species, including humans, depends on various physiologic conditions.

Substituting Eqs. 3 and 5 into $\Pi(t)=P(t) / P_{\text {iso }}(t)$ and applying the result of this into Eq. 25 finally yields:

$$
\begin{aligned}
\frac{d \epsilon}{d t} & =\beta \cdot(1+\sigma) \cdot\left[1+\frac{B_{e}}{B_{t}-B_{d}}\right] \cdot S\left\{\frac{d \phi}{d t}\right\} \\
\frac{d B_{e}}{d t} & =-\frac{\epsilon+\sigma}{\mu} \cdot B_{e}+\frac{\sigma}{\mu} \cdot\left(B_{t}-B_{d}\right) .
\end{aligned}
$$

The function $S(f)$ being defined as a multiplication by the value of its argument if this is positive, otherwise by zero.

Equations 27 and 28 constitute the description of an alinear state model of the left ventricle with state variables $B_{e}$ and $\epsilon$. Output $P(t)$ can be determined from the state variables by (3). The states are controlled by inputs $B_{t}-B_{d}$ and $d \phi / d t$. Parameter $\beta$ is a candidate for investigation regarding its clinical significance. Identification of alinear state models, currently, is an area of intensive study in systems engineering. The field has been reviewed by Nijmeijer and Van der Schaft (72).

\section{REFERENCES}

1. Abbot, B.C.; Mommaerts, W.F.H.M. A study of inotropic mechanisms in the papillary muscle preparation. J. Gen. Physiol. 42:533-551; 1959.

2. Abel, F.L. Fourier analysis of the left ventricular performance: Evaluation of impedance matching. Circ. Res. 28:119-135; 1971.

3. Anderson, P.A.W.; Manring, A.; Server, G.A.; Benson, D.W.; Edwards, S.B.; Armstrong, B.E.; Sterba, R.J.; Floyd, R.D. The force interval relationship of the left ventricle. Circ. Res. 47:334-348; 1979.

4. Arena, D.J.; Ohley, W.J. Analysis of left ventricular mechanics during filling, isovolumic contraction, and ejection. IEEE Trans. Biomed. Eng. 30:35-42; 1983. 
5. Armour, J.A.; Randall, W.C. Structural basis for cardiac function. Am. J. Physiol. 218:1517-1523; 1970.

6. Arts, T., Veenstra, P.C.; Reneman, S. A model of the mechanics of the left ventricle. Ann. Biomed. Eng. 7:299-318; 1979.

7. Avanzolinei, G.P.; Barbini, P.; Cappello, A.; Cevese, A. Time varying mechanical properties of the left ventricle - A computer simulation. IEEE Trans. Biomed. Eng. 32:756-763; 1985.

8. Bann, J.; Van Der Velde, T. Sensitivity of left ventricular end-systolic pressure-volume relation to type of loading intervention in dogs. Circ. Res. 62:1247-1258; 1988.

9. Bagni, M.M.A.; Cecchi, G.; Schoenberg, M. A model of force production that explains the lag between crossbridge attachment and force after electrical stimulation of striated muscle fibers. Biophys. J. 54:1105-1114; 1988.

10. Beyar, R.; Sideman, S. A model for left ventricular contraction combining the force length velocity relationship with the time varying elastance theory. Biophys. J. 45:1167-1177; 1984 .

11. Beyar, R.; Sideman, S. Computer studies of left ventricular performance based on its fiber structure sarcomere dynamics and transmural electrical activation propagation. Circ. Res. 55:358-375; 1984.

12. Beyar, R.; Sideman, S. Source parameters of the left ventricle related to the physiological characteristics of the cardiac muscle. Biophys. J. 19:1185-1194; 1986.

13. Boom, H.B.K. Elasticity of the heart. Utrecht, The Netherlands; 1972. PhD Dissertation.

14. Boom, H.B.K.; Denier van der Gon, J.J.; Nieuwenhuijs, J.H.M.; Schiereck, P. Cardiac Contractility: Actin-myosin interaction as measured from the left ventricular pressure curve. Europ. J. Cardiol. 1/2:217-224; 1973.

15. Boom, H.B.K.; Wijkstra, H. Identification of left ventricular model parameters. Proc. Ann. Int. Conf. of the IEEE Engineering in Medicine and Biology Society. Seattle, WA; 1989: Vol. 11, pp. 126127.

16. Brady, A.J. Time and displacement dependency of cardiac contractility: Problems in defining the active state and force-velocity relations. Fed. Proc. 24: pp. 1410; 1965.

17. van der Broek, J. A model study of left ventricular function. Utrecht, The Netherlands; 1979. PhD dissertation.

18. Brutsaert, D.L.; De Clerck, N.M.; Goethals, M.A.; Housmans, P.R. Relaxation of ventricular cardiac muscle. J. Physiol. 283:469-480; 1978.

19. Burkhoff, D.; Sugiura, S.; Yue, D.T.; Sagawa, K. Contractility dependent curvilinearity of endsystolic pressure-volume relations. Am. J. Physiol. 252:H1218-H1227; 1987.

20. Campbell, K.B.; Ringo, J.A.; Wakao, Y.; Klavano, A.; Alexander, J.E. Internal capacitance and resistance allow prediction of right ventricle outflow. Am. J. Physiol. 243:H99-H112; 1982.

21. Campbell, K.B.; Ringo, J.A.; Nebi, C.; Alexander, J.E. Informational analysis of left ventricle/systemic arterial interaction, Ann. Biomed. Eng. 12:209-231; 1984.

22. Campbell, K.B.; Ringo, J.A.; Knowlen, G.G.; Kirkpatrick, R.D.; Schmidt, S.L. Validation of optional elastance-resistance left ventricle pump models. Am. J. Physiol. 251:H382-H397; 1986.

23. Campbell, K.B.; Bell, D.B.; Ringo, J.A. Kinetic models for pressure response to volume change in tetanized isolated heart. Proc. of the Ann. Int. Conf. of the IEEE Eng. in Med. and Biol. Society. Seattle, WA; 1989: Vol. 11, pp. 120-121.

24. Chiu, Y.C.; Ballou, E.W.; Ford, L.E. Force, velocity, and power changes during normal and potentiated contractions of cat papillary muscle, Circ. Res. 60;446-458; 1987.

25. Clark, J.W.; Pruett, R.C.; Baldridge, D.L.; Srinivasan, R.; Bourland, H.M.; Cole, J.S.; Brower, R.W. Functional model for the characterization of the ventricular mechanics of the human subject, Med. Biol. Eng. Comp. 15:335-338; 1977.

26. Clark Jr., J.W.; Ling, R.Y.S.; Srinivasan, R.; Cole, J.S.; Purett, R.C. A two-stage identification scheme for the determination of the parameters of a model of left heart and systemic circulation. IEEE Trans. Biomed. Eng. 27:20-29; 1980.

27. Covell, J.W.; Taylor, R.R.; Sonnenblick, E.H.; Ross Jr., J. Series-elasticity of the Hill model for muscle applied to the intact left ventricle. Pfluegers Arch. 357:225-236; 1975.

28. Crozatier, B.; Caillet, D.; Thuillez, C.; Chevrier, J.L.; Peronneau, P.; Hatt, P.Y. Preload changes modify systolic pressure-diameter relations in the conscious dog. Am. J. Physiol. 240:H354-H360; 1981.

29. Deswijsen, B.A. Parameter of a simple model of the left ventricle and of the systemic vascular bed, with particular attention to the physical meaning of the left ventricular parameters. IEEE Trans. Biom. Eng. BME-24:29-39; 1977. 
30. Dieudonne, J.M. The left ventricle as confocal prolate spheroids. Bull. Math. Biophys. 31:433-439; 1969.

31. Ducas, J.; Schick, U.; Girling, L.; Prewitt, R.M. Effects of reduced resistive afterload on left ventricular pressure-volume relations. Am. J. Physiol. 248:H163-H169; 1985.

32. Edman, K.A.P.; Nilsson. E. The mechanical parameters of myocardial contraction studied at constant length of the contractile element. Acta. Physiol. Scand. 72:205-219; 1968.

33. Edman, K.A.P.; Nilsson, E. Relatinships between force and velocity of shortening in rabbit papillary muscle. Acta. Physiol. Scand. 85:488-500; 1972.

34. Eisenberg, E.; Hill, T.L. A cross-bridge model of muscle contraction. Prog. Biophys. Mol. Biol. $33: 55-82 ; 1978$.

35. Eisenberg, E.; Hill, T.L.; Chen, Y. Cross-bridge model of muscle contraction; Quantitative analysis. Biophys. J. 29:195-227; 1980.

36. Eisenberg, E.; Greene, L.E. The relation of muscle biochemistry to muscle physiology. Ann. Rev. Physiol. 42:293-309; 1980.

37. Elzinga, G.; Westerhof, N. "Pressure-volume" relations in isolated cat trabecula. Circ. Res. 49:388394; 1981.

38. Fabiato, A.; Fabiato, F. Dependence of the contractile activation of skinned cardiac cells on the sarcomere length. Nature Lond. 256:54-56; 1975.

39. Frank, O. Die Grundform des arteriellen pulses. Z. Biol. 37:483-526; 1898.

40. Gault, J.H.; Ross, J.; Braunwald, E. Contractile state of the left ventricle in man: Instantaneous tension-velocity-length relations in patients with and without disease of the left ventricular myocardium. Circ. Res. 22: p. 451, 1968.

41. Gould, P.; Ghista, D.; Brombolich, L. In vivo stresses in the human left ventricular wall: Analysis accounting for the irregular 3-dimensional geometry and comparison with idealized geometry analyses. J. Biomech. 5:521-539; 1972.

42. Granzier, H.L.M.; Pollack, G.H. Stepwise shortening in unstimulated skeletal muscle fibers. J. Physiol. 362:173-188; 1985.

43. Grossman, W.; Braunwald, E.; Mann, T.; McLaurin, L.P.; Green, L.H. Contractile state of the left ventricle in man as evaluated from end-systolic pressure-volume relations. Circulation 56:845-852; 1977.

44. Hibberd, M.G.; Jewell, B.R. Calcium and length dependent force production in rat ventricular muscle. J. Physiol. 333:173-188; 1982.

45. Hill, T.L. Theoretical formalism for the sliding filament model of contraction of striated muscle, Part I. Prog. Biophys. Mol. Biol. 28:267-340; 1974.

46. Hood, Jr., W.P.; Thomson, W.J.; Rackley, C.E.; Rolett, E.L. Comparison of calculations of left ventricular wall stress in man from thin-walled and thick-walled ellipsoidal models. Circ. Res. 24:575$582 ; 1969$.

47. Hunter, W.C.; Janicki, J.S.; Weber, K.T. Mechanical properties of the ventricle during systole. Federation Proc. 39:169-174; 1980.

48. Hunter, W.C.; Janicki, J.S.; Weber, K.T.; Noordergraaf, A. Flow-pulse response: A new method for the characterization of ventricular mechanics. Am. J. Physiol. 237:H282-H292; 1979.

49. Huxley, A.F. Muscle structure and theories of contraction. Prog. Biophys. Chem. 7:255-318; 1957.

50. Huxley, A.F.; Simmons, R.M. Proposed mechanism of force generation in striated muscle. Nature 233:533-538; 1971.

51. Huxley, A.F. Muscular contraction. J. Physiol. 243:1-43; 1974.

52. Igarashi, Y.; Goto, Y.; Yamada, O.; Ishii, T.; Suga, H. Transient vs. steady end-systolic pressurevolume relation in dog left ventricle. Am. J. Physiol. 252:H998-H1004; 1987.

53. Isaaz, K.; Pasipoularides, A. Noninvasive assessment of intrinsic ventricular load dynamics in dilated cardiomyopathy. J. Am. Coll. Cardiol. 17:112-21; 1991.

54. Janz, R.F.; Grimm, A.F. Finite-element model for the mathematical behaviour of the left ventricle. Circ. Res. 30:224-252; 1972.

55. Jewell, B.R. A reexamination of the influence of muscle length on myocardial performance. Circ. Res. 40:221-230; 1977.

56. Julian, F.J.; Sollins, K.R.; Sollins, M.R. A Model for the transient and steady-state mechanical behavior of contracting muscle. Biophys. J. 14:546-562; 1974.

57. Kaseda, K.H.; Tomoike, H.; Ogata, I.; Nakamura, M. End systolic pressure-volume, pressure-length, and stress-strain relations in canine hearts. Am. J. Physiol. 249:H648-H654; 1985. 
58. Kass, D.A.; Maughan, W.L. From "Emax" to pressure-volume relations: A broader view. Circulation 77:1203-1212; 1988 .

59. Kaufmann, R.L.; Bayer, R.M.; Harnasch, C. Autoregulation of contractility in the myocardial cell. Pfluegers Arch. 332:96-116; 1972.

60. Keurs, H.E.D.J. ter; Rijnsburger, W.H.; van Heuningen, R., Nagelsmit, M.J. Tension development and sarcomere length in rat cardiac muscle. Circ. Res. 46:703-714; 1980.

61. Kil, P.J.M.; Schiereck, P. Influences of the velocity of changes in end-diastolic volume on the starling mechanism of isolated left ventricles. Pfluegers Arch. 396:243-253; 1983.

62. Kil, P.J.M.; Schiereck, P. End-systolic pressure-volume relations of isolated ejecting rabbit left ventricles after quick-diastolic volume changes. Cardiovasc. Res. 19:782-792; 1985.

63. Little, W.C. The left ventricular $d P / d t_{\max }$-end diastolic volume relation in closed-chest Dogs. Circ. Res. 56:808-815; 1985.

64. Maughan, W.L.; Sunagawa, K.; Burkhof, D.; Sagawa, K. Effect of arterial impedance changes on the end-systolic pressure-volume relation. Circ. Res. 54:595-602; 1984.

65. McHale, P.A.; Greenfield, J.C. Evaluation of several geometric models for estimation of left ventricular circumferential wall stress. Circ. Res. 33:303-312; 1973.

66. Mirsky, I. Ventricular and arterial wall stresses based on large deformation analysis. Biophys. J. 13:1141-1159; 1973.

67. Monroe, G.; French, G.M. Left ventricular pressure-volume relationships and myocardial oxygen consumption in isolated heart. Circ. Res. 9:362-374; 1961.

68. Nead, N.S.; Klein, M.D.; Mirsky, I.; Lown, B. Assessment of myocardial contractility from ventricular pressure recordings. Cardiovasc. Res. 5:15-23; 1971.

69. Negroni, J.A.; Lascano, E.C.; Pichel, R.H. Relationship between chamber mechanical properties and mean pressure-mean flow diagram of the left ventricle. Ann. Biomed. Eng. 15:35-50; 1987.

70. Negroni, J.A.; Lascano, E.C.; Pichel, R.H. A computer study of the relation between chamber mechanical properties and mean pressure-mean flow of the left ventricle. Circ. Res. 62:1121-1133; 1988.

71. Nelder, J.A.; Mead, R. A simplex method for function minimization. The Computer Journal 7:308$313 ; 1965$.

72. Nijmeijer, H.; Van der Schaft, A.J. Nonlinear dynamical control systems. New York: Springer-Verlag; 1990.

73. Nwasokwa, O.; Sagawa, K.; Suga, H. Short-term memory in the in situ canine myocardium. Am. J. Physiol. 247:H8-H16; 1984.

74. Pao, Y.C.; Ritman, E.L.; Wood, E.H. Finite element analysis of left ventricular myocardial stresses. J. Biomech. 7:469-477; 1974.

75. Pasipoularides, A. Clinical assessment of ventricular ejection dynamics with and without outflow obstruction. J. Am. Coll. Cardiol. 15:859-82; 1990.

76. Pasipoularides, A.; Murgo, J.P.; Miller, J.W.; Craig, W.E. Nonobstructive left ventricular ejection pressure gradients in man. Circ. Res. 61:220-227; 1987.

77. Pasipoularides, A.; Murgo, J.P.; Bird, J.J.; Vraig, W.E. Fluid dynamics of aortic stenosis: Mechanisms for the presence of subvalvular pressure gradients. Am. J. Physiol. 246:H542-H550; 1984.

78. Pollack, G.H. Quantal mechanisms in cardiac contraction. Circ. Res. 59:1-8; 1986.

79. Rankin, J.S.; McHale, P.; Arentzen, C.E.; Ling, D.; Greenfield, J.C.; Anderzon, F.W. The threedimensional dynamic geometry of the left ventricle in the conscious dog. Circ. Res. 39:304-313; 1976.

80. Regen, D.M. Relations between hydrodynamics and mechanical properties of a sphere. Ann Biomed. Eng. 16:573-588; 1988.

81. Regen, D.M. Effect of chamber shape and fiber orientation on relations between fiber dynamics and chamber dynamics. Ann. Biomed. Eng. 16:589-607; 1988.

82. Reichel, H. Die Beziehungen zwischen laenge und spannung, volumen und druck. Z. Biol. 99:63$79 ; 1939$.

83. Sagawa, K.; Suga, H.; Shoukad, A.A.; Bakalar, K.M. End-systolic pressure-volume ratio: A new index for contractility. Am. J. Cardiol. 40:748-753; 1977.

84. Sarnoff, S.J.; Mitchell, J.H.; Gilmore, J.P.; Remensnyder, J.P. Homeometric autoregulation in the heart. Circ. Res. 8:1077-1091; 1960.

85. Schiereck, P.; Boom, H.B.K. Left ventricular active stiffness: Dependency on time and inotropic state. Pfluegers Arch. 374:135-143; 1978.

86. Schiereck, P.; Boom, H.B.K. Left ventricular force-velocity relation measured from quick volume changes. Pfluegers Arch. 379:251-258; 1979. 
87. Shroff, S.G.; Janicki, J.S. Weber, K.T. Evidence and quantification of left ventricular systolic resistance. Am. J. Physiol. 249:H358-H370; 1985.

88. Slinker, B.K.; Goto, Y.; Le Winter, M.M. Systolic direct ventricular interaction affects left ventricular contraction and relaxation in the intact dog circulation. Circ. Res. 65:307-315; 1989.

89. Sonnenblick, E.H. Force velocity relations in mammalian heart muscle. Am. J. Physiol. 202:931939; 1962.

90. Spotnitz, H.M.; Sonnenblick, E.H.; Spiro, D. Relation of ultrastructure to function in intact heart. Sarcomere structure relative to pressure-volume curves of intact left ventricles of dog and cat. Circ. Res. 18:49-66; 1966.

91. Spratt, J.A.; Tyson, G.S.; Glower, D.D.; Davis, J.W.; Muhlbaier, L.H.; Olsen, C.O.; Rankin, J.S. The end-systolic pressure-volume relation in conscious dogs. Circulation 75:1295-1309; 1987.

92. Streeter, D.D.; Spotnitz, H.M.; Patel, D.P.; Ross, J.; Sonnenblick, E.H. Fiber orientation in the canine left ventricle during diastole and systole. Circ. Res. 33:656-664; 1973.

93. Suga, H. Analysis of left ventricular pumping by its pressure volume coefficient. Jap. J. Med. Electronics Biol. Eng. 7:406-415; 1969.

94. Suga, H.; Sagawa, K. Mathematical interrelationship between instantaneous ventricular pressurevolume ratio and myocardial force-velocity relation. Ann. Biomed. Eng. 1:160-181; 1972.

95. Suga, H. Sagawa, K.; Shoukas, A.A. Load independence of the instantaneous pressure-volume ratio of the canine left ventricle and effects of epinephrine and heart rate on the ratio. Circ. Res. 32:314322; 1973.

96. Suga, H.; Sagawa, K. Instantaneous pressure-volume relationships and their ratio in the excised, supported canine left ventricle. Circ. Res. 35:117-126; 1974.

97. Suga, H.; Sagawa, K.; Demer, L. Determinants of instantaneous pressure in canine left ventricle: Time and volume specification. Circ. Res. 46:256-263; 1977.

98. Taylor, R.R.; Covell, J.W.; Ross, Jr., J. Volume-tension diagrams of ejection and isovolumic contractions in left ventricle. Am. J. Physiol. 216:1097-1102; 1969.

99. Templeton, G.H.; Ecker, P.R.; Mitchell, J.H. Left ventricular stiffness during diastole and systole: The influence of changes in volume and inotropic state. Cardiovasc. Res. 6:95-100; 1972.

100. Ullrich, K.J.; Riecker, G.; Kramer, K. Das druckvolumendiagramm des warmblueterherzens. Pfluegers Arch. 259: p. 481; 1954

101. Vaartjes, S.R.; van Alste, J.A. Identifications of the hydraulic source parameters of the left ventricle of the heart (abstract). Proc. World Congress on Medical Physics and Biomedical Engineering. Hamburg, Germany, 1982.

102. Vaartjes, S.R.; Boom, H.B.K. Left ventricular internal resistance and unloaded ejection flow assessed from pressure-flow relations: A flow-clamp study on isolated rabbit hearts. Circ. Res. 60:727-737; 1987.

103. Voukydis, P.C. Physiologic significance of the geometrical shape of the left ventricle: Course and curvature of the individual myocardial fibers. Bull. Math. Biophys. 31:383; 1969.

104. Waldman, L.K.; Nosan, D.; Villareal, F.; Covell, J.W. Relation between transmural deformation and local myofiber direction in canine left ventricle. Circ. Res. 63:550-562; 1987.

105. Weber, K.T.; Janicki, J.S. The dynamics of ventricular contraction. Federation Proc. 39:188-195; 1980.

106. Weber, K.T.; Janicki, J.S.; Hunter, W.C.; Shroff, S.; Pearlman, E.S.; Fishman, A.P. The contractile behavior of the heart and its functional coupling to the circulation. Prog. Cardiovasc. Dis. 24:375400; 1982.

107. Welkowitz, W. Indices of cardiac status. IEEE Trans. Biomed. Eng. BME-28:553-567; 1981.

108. Westerhof, N.; Elzinga, G. The apparent source impedance of heart and muscle. Ann. Biomed. Eng. $6: 16-32 ; 1978$.

109. Wijkstra, H.; Boom, H.B.K. Deactivation in the rabbit ventricle induced by constant ejection flow. IEEE Trans. Biomed. Eng. 36:1113-1123; 1989.

110. Wijkstra, H.; Boom, H.B.K. Left ventricular dynamic model based on constant ejection flow periods. IEEE Trans. Biomed. Eng. BME-38; 1989.

111. Wong, A.Y.K.; Rautaharju, P.M. Stress distribution within the left ventricular wall approximated as a thick-walled ellipsoidal shell. Am. Heart J. 75:649-661; 1968.

112. Yasumura, Y.; Nozawa, T.; Futaki, S.; Tanaka, N.; Suga, H. Time-invariant oxygen cost of mechanical energy in dog left ventricle: Consistency and inconsistency of time-varying elastance model with myocardial energetics. Circ. Res. 64:764-778; 1989. 